\title{
The excess of cool supergiants from contemporary stellar evolution models defies the metallicity-independent Humphreys-Davidson limit
}

\author{
Avishai Gilkis ${ }^{1 \star}$, Tomer Shenar ${ }^{2} \dagger$, Varsha Ramachandran ${ }^{3}$, Adam S. Jermyn ${ }^{4}$, \\ Laurent Mahy ${ }^{2}$, Lidia M. Oskinova ${ }^{3}$, Iair Arcavi ${ }^{1,5}$ and Hugues Sana ${ }^{2}$ \\ ${ }^{1}$ The School of Physics and Astronomy, Tel Aviv University, Tel Aviv 6997801, Israel \\ ${ }^{2}$ Institute of Astrophysics, KU Leuven, Celestijnenlaan 200 D, 3001 Leuven, Belgium \\ ${ }^{3}$ Institut für Physik und Astronomie, Universität Potsdam, Karl-Liebknecht-Str. 24 / 25, D-14476 Potsdam, Germany \\ ${ }^{4}$ Center for Computational Astrophysics, Flatiron Institute, 162 5th Ave, New York, NY 10010, USA \\ ${ }^{5}$ CIFAR Azrieli Global Scholars program, CIFAR, Toronto, Canada
}

8 February 2021

\begin{abstract}
The Humphreys-Davidson (HD) limit empirically defines a region of high luminosities $\left(\log _{10}\left(L / \mathrm{L}_{\odot}\right) \gtrsim 5.5\right)$ and low effective temperatures $\left(T_{\text {eff }} \lesssim 20 \mathrm{kK}\right)$ on the Hertzsprung-Russell Diagram in which hardly any supergiant stars are observed. Attempts to explain this limit through instabilities arising in near- or super-Eddington winds have been largely unsuccessful. Using modern stellar evolution we aim to re-examine the HD limit, investigating the impact of enhanced mixing on massive stars. We construct grids of stellar evolution models appropriate for the Small and Large Magellanic Clouds (SMC, LMC), as well as for the Galaxy, spanning various initial rotation rates and convective overshooting parameters. Significantly enhanced mixing apparently steers stellar evolution tracks away from the region of the HD limit. To quantify the excess of over-luminous stars in stellar evolution simulations we generate synthetic populations of massive stars, and make detailed comparisons with catalogues of cool $\left(T_{\text {eff }} \leq 12.5 \mathrm{kK}\right)$ and luminous $\left(\log _{10}\left(L / \mathrm{L}_{\odot}\right) \geq 4.7\right)$ stars in the SMC and LMC. We find that adjustments to the mixing parameters can lead to agreement between the observed and simulated red supergiant populations, but for hotter supergiants the simulations always over-predict the number of very luminous $\left(\log _{10}\left(L / \mathrm{L}_{\odot}\right) \geq 5.4\right)$ stars compared to observations. The excess of luminous supergiants decreases for enhanced mixing, possibly hinting at an important role mixing has in explaining the HD limit. Still, the HD limit remains unexplained for hotter supergiants.
\end{abstract}

Key words: stars: evolution - stars: massive

\section{INTRODUCTION}

The upper-right part of the Hertzsprung-Russell diagram (HRD) features a stark absence of observed stars (Fig. 1), a phenomenon termed the Humphreys-Davidson (HD) limit (Humphreys \& Davidson 1979). While the luminous blue variables (LBVs) venture into this region during outbursts, with a few exceptions, cool supergiants (CSGs), which comprise red, yellow, and blue supergiants (RSGs, YSGs, BSGs) with effective temperatures $T_{\text {eff }} \lesssim 12.5 \mathrm{kK}$ and luminosities $\log _{10}\left(L_{\max } / \mathrm{L}_{\odot}\right) \gtrsim 5.5$ (i.e., initial masses $\left.M_{\mathrm{i}} \gtrsim 30 \mathrm{M}_{\odot}\right)$ are not observed. In contrast, hundreds of main sequence progenitors with $M_{\mathrm{i}} \gtrsim 30 \mathrm{M}_{\odot}$, ranging all the way to $\approx 150 \mathrm{M}_{\odot}$ and perhaps more, were directly observed (Crowther et al. 2010; Bestenlehner et al. 2011; Almeida et al. 2017; Shenar et al. 2017; Tehrani et al. 2019; Mahy et al. 2020). This implies two possibilities: either stars with $M_{\mathrm{i}} \gtrsim 30 \mathrm{M}_{\odot}$ skip the CSG phase altogether, or they experience this phase very briefly, making it observationally rare.

Despite various attempts, no evolutionary models are currently ca-

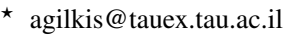

$\dagger$ tomer.shenar@kuleuven.be
}

pable of reproducing the observed absence of stars beyond the HD limit (Davies, Crowther \& Beasor 2018; Schootemeijer \& Langer 2018). The HD limit has consequences not only for our understanding of the evolution of the progenitors of Wolf-Rayet (WR) stars and black holes (BHs), but also for estimates of the likelihood of binary interaction in the upper-mass end of stars. The reliability of our predictions of gravitational-wave (GW) events are thus severely limited as long as the HD limit has not been sufficiently understood.

It is commonly assumed that the HD limit is a result of radiative instability, tracing a region in which stars reach their Eddington luminosity and become LBVs (e.g., Lamers \& Fitzpatrick 1988; Glatzel \& Kiriakidis 1993). However, attempts to prove this by means of direct radiative transfer calculations in stellar models have had only limited success. Ulmer \& Fitzpatrick (1998) showed that the so-called modified Eddington limit mimics the shape of the HD limit, but that it lies roughly one magnitude above the observed HD limit. More recently, Sanyal et al. (2017) showed that stars with $M_{\mathrm{i}} \gtrsim 30 \mathrm{M}_{\odot}$ and a solar metallicity content $\left(Z=Z_{\odot}\right)$ reach the Eddington limit in their interiors and undergo envelope inflation. However, both these studies and others clearly predict that $L_{\max }$ should grow with decreasing $Z$. This is because the opacities in the inte- 

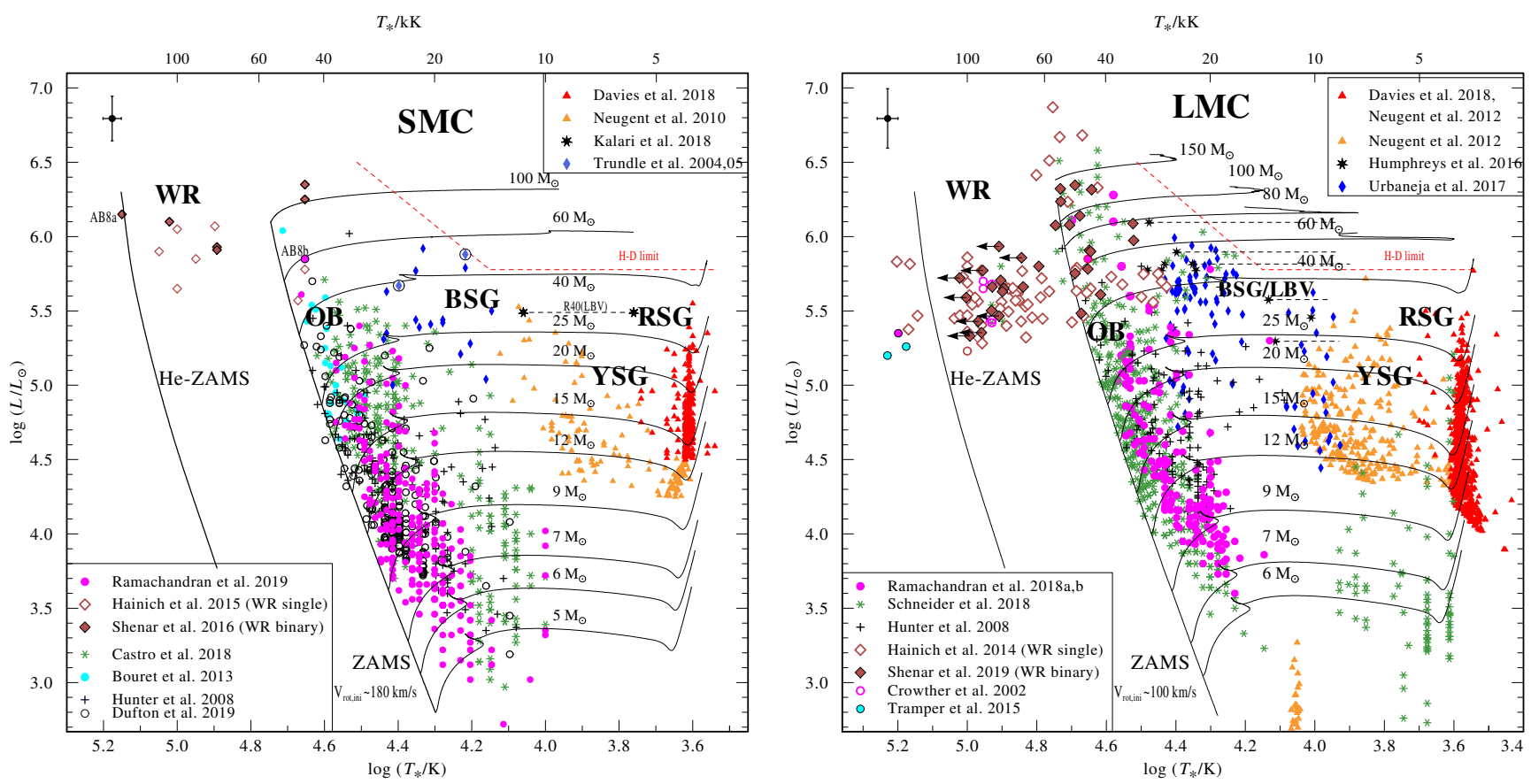

Figure 1. HRD positions of populations of massive stars in the SMC (left panel) and LMC (right panel), based on analyses of apparently single and binary WR stars (Hainich et al. 2014, 2015; Shenar et al. 2016, 2019; Crowther et al. 2002; Tramper et al. 2015), YSGs (Neugent et al. 2010, 2012), RSGs (Davies et al. 2018; Neugent et al. 2012), LBVs (Humphreys et al. 2016; Kalari et al. 2018), BSGs (Trundle et al. 2004; Trundle \& Lennon 2005; Urbaneja et al. 2017), and populations of OB-type stars (Ramachandran et al. 2018a,b, 2019; Schneider et al. 2018; Hunter et al. 2008; Castro et al. 2018; Dufton et al. 2019; Bouret et al. 2013). The typical error bar is shown in the top left corner. Evolutionary tracks (black solid lines), accounting for rotation with $v_{\text {rot,init }} \approx 100 \mathrm{~km} \mathrm{~s}^{-1}$ for the LMC and $\approx 180 \mathrm{~km} \mathrm{~s}^{-1}$ for the SMC (Brott et al. 2011; Köhler et al. 2015), are labeled with their initial mass. The red dashed lines mark the observational limit reported by Humphreys \& Davidson (1979). The diagrams are largely complete for supergiants with $T_{\text {eff }} \lesssim 12.5 \mathrm{kK}$, but not necessarily complete for hotter stars.

rior of stars are strongly correlated with the content of metals within them. The lower $Z$ is, the weaker the outward radiative pressure becomes. Hence, from this perspective, environments with a lower ambient $Z$ are expected to allow the stability of more massive stars, and higher $L_{\max }$.

This prediction does not seem to be confirmed by observations. Humphreys \& Davidson (1979) originally reported a maximum RSG luminosity at around $\log _{10}\left(L / \mathrm{L}_{\odot}\right) \approx 5.7$, later revised slightly downwards to 5.6 by Levesque et al. (2005). Similar values are reported by Massey \& Evans (2016) and Drout, Massey \& Meynet (2012) for the super-solar metallicity environment of the Andromeda galaxy (M31) and the approximately solar $Z$-environment of the Triangulum galaxy (M33).

This trend continues with the Small and Large Magellanic Clouds (SMC, LMC), which have metallicities of $Z \approx 0.15,0.4 Z_{\odot}$, respectively (Korn et al. 2000; Hunter et al. 2007; Trundle et al. 2007). Figure 1 shows the HRD positions of analysed massive stars in the SMC and LMC, adapted from Ramachandran et al. (2018b, 2019). These populations are thought to be complete for the WRs as well as the luminous $\left(\log _{10}\left(L / L_{\odot}\right) \gtrsim 4.7\right)$ YSGs and RSGs, but far from complete for the OB-type stars. Figure 1 illustrates strongly the absence of RSGs with $\log _{10}\left(L / \mathrm{L}_{\odot}\right) \gtrsim 5.5$ in both galaxies, with the exception of two peculiar objects in the LMC: HD 33579 (Wolf 1972) and WOH G 64 (Ohnaka et al. 2008).

Davies et al. (2018) performed a statistical comparison of analyzed RSG populations in the SMC and LMC, and showed compelling evidence that $\log _{10}\left(L_{\max } / \mathrm{L}_{\odot}\right) \lesssim 5.5$ in both galaxies. Moreover, no evidence is found for higher $L_{\max }$ in the SMC compared to the LMC. Davies et al. (2018) illustrated why this is very likely a genuine physical fact rather than a statistical one, and how this stands in tension with recent rotating and non-rotating Geneva evolution models (Ekström et al. 2012; Georgy et al. 2013).

The challenge of explaining the HD limit solely by radiative instabilities might imply that other processes are involved. It was already recognized in the past that rotationally induced mixing can strongly hinder the redward evolution of massive stars towards the RSG phase (e.g., Maeder \& Meynet 2000). However, initial rotation rates in the excess of $\approx 200 \mathrm{~km} \mathrm{~s}^{-1}$ (at which strong rotational mixing starts) seem to be inconsistent with observed rotation rates of massive stars (Ramírez-Agudelo et al. 2015, 2017; Ramachandran et al. 2019), and models using realistic rotation rates do not reproduce the apparently $Z$-independent HD limit (Davies et al. 2018).

Higgins \& Vink (2020) recently explored this problem by investigating the impact of mixing parameters on RSG stellar models. They found that enhanced semi-convection can reproduce the observed HD limit. However, a solution to the HD limit problem needs to consider YSGs and cool BSGs as well, as we argue in our study.

In this paper we tackle the puzzle of the HD limit from a different angle, quantifying the duration spent beyond the HD limit and the expected number of stars for various stellar evolution models. For this purpose we study models with enhanced mixing and construct synthetic populations based on these models. Moreover, unlike previous studies that considered RSGs alone, we consider simultaneously RSGs, YSGs, and cool BSGs ( $T_{\text {eff }} \lesssim 12.5 \mathrm{kK}$ ). As our study shows, considering CSGs as a whole is vital for correctly assessing the discrepancy between models and observations.

The paper is organized as follows. In Section 2 we describe our numerical approach. In Section 3 we show the effect of enhanced 
Table 1. Initial compositions in terms of mass fractions for stellar evolution calculations.

\begin{tabular}{cccc}
\hline \hline & SMC & LMC & MW \\
\hline $\mathrm{H}$ & 0.75328 & 0.74392 & 0.72031 \\
$\mathrm{He}$ & 0.24448 & 0.25072 & 0.26646 \\
$\mathrm{C}$ & 0.00039621 & 0.00094807 & 0.0023401 \\
$\mathrm{~N}$ & 0.00011606 & 0.00027772 & 0.00068549 \\
$\mathrm{O}$ & 0.00096042 & 0.0022981 & 0.0056725 \\
$\mathrm{Ne}$ & 0.00021246 & 0.00050838 & 0.0012548 \\
$\mathrm{Mg}$ & 0.00011456 & 0.00027412 & 0.00067661 \\
$\mathrm{Si}$ & 0.00012179 & 0.00029144 & 0.00071934 \\
$\mathrm{~S}$ & 0.000056647 & 0.00013555 & 0.00033457 \\
$\mathrm{Ar}$ & 0.000013447 & 0.000032178 & 0.000079424 \\
$\mathrm{Ca}$ & 0.00001175 & 0.000028117 & 0.0000694 \\
$\mathrm{Fe}$ & 0.00023666 & 0.0005663 & 0.0013978 \\
\hline $\mathrm{Z}$ & 0.00224 & 0.00536 & 0.01323 \\
\hline
\end{tabular}

mixing on stellar evolution tracks. In Section 4 we make comparisons between observations and synthetic populations that we generate from our stellar evolution tracks. In Section 5 we examine the impact of modelling assumptions. We discuss our results in relation to previous studies, and summarise, in Section 6.

\section{NUMERICAL METHOD}

We use the Modules for Experiments in Stellar Astrophysics code (MESA, version 10398, Paxton et al. 2011, 2013, 2015, 2018) to evolve stellar models with 39 different zero-age main sequence (ZAMS) masses between $M_{\mathrm{ZAMS}}=4 \mathrm{M}_{\odot}$ and $M_{\mathrm{ZAMS}}=107 \mathrm{M}_{\odot}$. Three different initial compositions are used, appropriate for SMC, LMC and Milky Way (MW) stars, as listed in Table 1 (following Hainich et al. 2019).

\subsection{Microphysics}

The equation of state employed by MESA is a blend of the following equations of state: OPAL (Rogers \& Nayfonov 2002), SCVH (Saumon, Chabrier \& van Horn 1995), HELM (Timmes \& Swesty 2000), and PC (Potekhin \& Chabrier 2010). Radiative opacities are primarily from OPAL (Iglesias \& Rogers 1993, 1996), with low-temperature data from Ferguson et al. (2005) and the hightemperature, Compton-scattering dominated regime according to Buchler \& Yueh (1976). Electron conduction opacities follow Cassisi et al. (2007).

We use the built-in MESA nuclear reaction network approx21. The Joint Institute for Nuclear Astrophysics REACLIB reaction rates (Cyburt et al. 2010) are used, with additional tabulated weak reaction rates (Fuller, Fowler \& Newman 1985; Oda et al. 1994; Langanke \& Martínez-Pinedo 2000) and screening via the prescriptions of Salpeter (1954), Dewitt, Graboske \& Cooper (1973), Alastuey \& Jancovici (1978) and Itoh et al. (1979). The formulae of Itoh et al. (1996) are used for thermal neutrino loss rates.

\subsection{Wind mass loss}

For cool phases ( $\left.T_{\text {eff }} \leq 10000 \mathrm{~K}\right)$, the mass-loss prescription of de Jager, Nieuwenhuijzen \& van der Hucht (1988) is employed, regardless of the surface hydrogen mass fraction $X_{\mathrm{s}}$. Wind mass loss is according to Vink, de Koter \& Lamers (2001) for hot $\left(T_{\text {eff }} \geq 11000 \mathrm{~K}\right)$ hydrogen-rich phases $\left(X_{\mathrm{s}} \geq 0.4\right)$ of the evolution, most notably the main sequence. If the surface hydrogen mass fraction is low but non-negligible $\left(0.1 \leq X_{\mathrm{s}}<0.4\right)$ then the empirical mass-loss rate relation of Nugis \& Lamers (2000) is used. For hot hydrogendeficient models $\left(X_{\mathrm{s}}<0.1\right)$ we follow Yoon (2017) and Woosley (2019), whose prescriptions are based on the mass-loss rates of Hainich et al. (2014) and Tramper, Sana \& de Koter (2016). For $10000 \mathrm{~K}<T_{\text {eff }}<11000 \mathrm{~K}$ we interpolate between the two regimes described above. The mass-loss rate is multiplied by a factor $\eta_{\mathrm{w}}$. We mostly use $\eta_{\mathrm{w}}=1$, but in one set of models we explore the impact of boosted mass loss on our results by taking $\eta_{\mathrm{w}}=2$ (Section 5.1) throughout the entire evolution. We note that the metallicity dependence of the mass-loss rate during the main sequence, which follows Vink et al. (2001), is $\dot{M} \propto Z^{0.85}$. The fit by de Jager et al. (1988), which prevails during most of the CSG phases, has no implicit dependence on metallicity.

\subsection{Mixing and rotation}

The models have initial rotation velocities at the equator of $V_{\mathrm{i}}=100$, 200 , and $300 \mathrm{~km} \mathrm{~s}^{-1}$. The shellular approximation where the angular velocity is constant over isobars (Meynet \& Maeder 1997) is used for rotating models in MESA (Paxton et al. 2013). Mixing processes induced by rotation are implemented in a diffusion approximation (Paxton et al. 2013) and principally follow Heger, Langer \& Woosley (2000). Transport of angular momentum and chemical mixing caused by internal magnetic fields follows the new prescription of Fuller, Piro \& Jermyn (2019).

The efficiency of rotational mixing is set by two parameters, the ratio of the turbulent viscosity to the diffusion coefficient $f_{c}$ (input parameter am_D_mix_factor in MESA), and the ratio between the actual molecular weight gradient and the value used for computing the mixing coefficients $f_{\mu}$ (input parameter am_gradmu_factor in MESA). These parameters are calibrated to give the observed nitrogen enhancement for evolved stars. We use the calibration of Heger et al. (2000), $f_{c}=1 / 30$ and $f_{\mu}=0.05$ for most models. However, as discussed by Chieffi \& Limongi (2013), this calibration is not unique (also Potter, Tout \& Eldridge 2012). In one set of models we use alternative values of $f_{c}=0.2$ and $f_{\mu}=1$ (Chieffi \& Limongi 2013).

Convective regions are defined by the Ledoux criterion (except for one set of models which employs the Schwarzschild criterion) and treated according to Henyey, Vardya \& Bodenheimer (1965) with a mixing-length parameter of $\alpha_{\mathrm{MLT}}=1.5$. Semiconvective mixing in regions which are Ledoux stable but Schwarzschild unstable follows Langer, Fricke \& Sugimoto (1983) with an efficiency parameter $\alpha_{\mathrm{sc}}=1$ or $\alpha_{\mathrm{sc}}=100$. Thermohaline mixing is according to Kippenhahn, Ruschenplatt \& Thomas (1980) with an efficiency parameter of $\alpha_{\mathrm{th}}=1$. We use the so-called MLT ++ implementation for efficient energy transport in convective regions (Paxton et al. 2013).

Mixing above the convective core boundary is extended in two approaches. First, for most models, a step overshoot approach is taken (e.g. Shaviv \& Salpeter 1973; Maeder \& Meynet 1987), where the convective region is extended by a fraction $\alpha_{\mathrm{ov}}$ of the pressure scale height $H_{P}$. The lowest value for $\alpha_{\mathrm{ov}}$ that we use is $\alpha_{\mathrm{ov}}=0.1$ (e.g. Ekström et al. 2012; Higgins \& Vink 2020), followed by $\alpha_{\text {ov }}=0.335$ as calibrated by Brott et al. (2011), with higher values starting at $\alpha_{\mathrm{ov}}=0.5$ (e.g. Vink et al. 2010; Higgins \& Vink 2019), and ad- 
Table 2. Modelling assumptions for all sets of models computed.

\begin{tabular}{ccccccc}
\hline \hline$\#$ & $\begin{array}{c}\text { stability } \\
\text { criterion }\end{array}$ & overshooting & $\alpha_{\mathrm{sc}}$ & $f_{c}$ & $f_{\mu}$ & $\eta_{\mathrm{w}}$ \\
\hline 1 & Ledoux & $\alpha_{\mathrm{ov}}=0.1$ & 1 & $1 / 30$ & 0.05 & 1 \\
2 & Ledoux & $\alpha_{\mathrm{ov}}=0.335$ & 1 & $1 / 30$ & 0.05 & 1 \\
3 & Ledoux & $\alpha_{\text {ov }}=0.5$ & 1 & $1 / 30$ & 0.05 & 1 \\
4 & Ledoux & $\alpha_{\mathrm{ov}}=0.8$ & 1 & $1 / 30$ & 0.05 & 1 \\
5 & Ledoux & $\alpha_{\mathrm{ov}}=1.0$ & 1 & $1 / 30$ & 0.05 & 1 \\
6 & Ledoux & $\alpha_{\mathrm{ov}}=1.2$ & 1 & $1 / 30$ & 0.05 & 1 \\
7 & Ledoux & $f_{\text {ov }, \mathrm{JTC}}$ & 1 & $1 / 30$ & 0.05 & 1 \\
\hline 8 & Ledoux & $\alpha_{\mathrm{ov}}=0.1$ & 100 & $1 / 30$ & 0.05 & 1 \\
9 & Ledoux & $\alpha_{\mathrm{ov}}=0.335$ & 100 & $1 / 30$ & 0.05 & 1 \\
10 & Ledoux & $\alpha_{\mathrm{ov}}=0.5$ & 100 & $1 / 30$ & 0.05 & 1 \\
11 & Ledoux & $\alpha_{\mathrm{ov}}=0.8$ & 100 & $1 / 30$ & 0.05 & 1 \\
12 & Ledoux & $\alpha_{\mathrm{ov}}=1.0$ & 100 & $1 / 30$ & 0.05 & 1 \\
13 & Ledoux & $\alpha_{\mathrm{ov}}=1.2$ & 100 & $1 / 30$ & 0.05 & 1 \\
14 & Ledoux & $f_{\mathrm{ov}, \mathrm{JTC}}$ & 100 & $1 / 30$ & 0.05 & 1 \\
\hline 15 & Schwarzschild & $\alpha_{\mathrm{ov}}=0.335$ & - & $1 / 30$ & 0.05 & 1 \\
16 & Ledoux & $\alpha_{\mathrm{ov}}=0.335$ & 100 & 0.2 & 1.0 & 1 \\
17 & Ledoux & $\alpha_{\mathrm{ov}}=0.335$ & 100 & $1 / 30$ & 0.05 & 2
\end{tabular}

ditional higher values of $\alpha_{\mathrm{ov}}=0.8, \alpha_{\mathrm{ov}}=1$ and $\alpha_{\mathrm{ov}}=1.2^{1}$. In a second approach we use an exponential core overshooting (e.g. Herwig 2000), where the mixing efficiency decays smoothly outside the core, rather than dropping abruptly as in the step overshoot approach. We follow the prescription of Jermyn, Tout \& Chitre (2018) to compute the fraction $f_{\text {ov }}$ used for the decay scale,

$$
f_{\mathrm{ov}}=\frac{2}{\ln 150-2 \ln \left(v_{\mathrm{c}} / c_{\mathrm{s}}\right)+\left(\frac{5}{2}\left(H_{P} / r_{\mathrm{c}}\right)-1\right)},
$$

where $v_{\mathrm{c}} / c_{\mathrm{s}}$ is the mass-averaged convective core Mach number, $H_{P}$ is taken at the top of the core, and $r_{\mathrm{c}}$ is the convective core radius. The value of $f_{\text {ov }}$ is updated according to equation (1) at the end of every evolution step. This prescription corresponds to a meridional circulation driven by anisotropy in the heat flux emerging from the convective core. The anisotropy is rotationally-induced, but because the convective turnover time is so long in the core this effect saturates at slower angular velocities than any we consider here.

\section{STELLAR EVOLUTION TRACKS}

A total of 5967 models were evolved, for 39 initial masses $\left(M_{\text {ZAMS }}=\right.$ $4,5,6,7,8,9,10,11,12,13,14,15,16,17,19,20,22,23,25$, $27,29,31,33,36,39,42,45,48,52,56,60,64,69,74,80,86$, 92 , 99, and $\left.107 \mathrm{M}_{\odot}\right), 3$ initial rotation velocities $\left(V_{\mathrm{i}}=100,200\right.$, and $300 \mathrm{~km} \mathrm{~s}^{-1}$ ), 3 compositions (SMC, LMC, and MW), and 17 sets of modelling assumptions as detailed in Table 2 . The evolution reached the end of core helium burning for the lower masses $\left(M_{\text {ZAMS }}<10 \mathrm{M}_{\odot}\right)$ or core carbon burning for the higher masses $\left(M_{\text {ZAMS }} \geq 10 \mathrm{M}_{\odot}\right)$. We present several sub-sets of the results to highlight our main findings.

Figure 2 shows evolutionary tracks for LMC and MW compositions and an initial rotation velocity of $V_{\mathrm{i}}=200 \mathrm{~km} \mathrm{~s}^{-1}$, for $\alpha_{\mathrm{sc}}=100$ and step overshooting with $\alpha_{\mathrm{ov}}=0.335$ and $\alpha_{\mathrm{ov}}=1.2$. Points at intervals of $50000 \mathrm{yr}$ along the evolution are marked with

1 These parameter values might not be physical, but rather used as proxy to investigate the issue at hand. dots, to illustrate the duration of different phases, and the relative expected number of stars at each part of the evolution for each initial mass. It can be seen that for $\alpha_{\mathrm{ov}}=0.335$ numerous models spend a long time beyond the HD limit, while for $\alpha_{\mathrm{ov}}=1.2$ the evolutionary tracks almost do not cross the limit, and then only for a brief time if they do. The value of $\alpha_{\mathrm{ov}}=1.2$ is probably excessive, as a gap is present between the tracks and the diagonal part of the HD limit. We do not suggest that $\alpha_{\mathrm{ov}}=1.2$ is a reasonable choice, but rather use it to demonstrate the strong effect of overshooting. To test the models a statistical analysis is discussed in Section 4.

Figure 3 shows evolutionary tracks for LMC and MW compositions and an initial rotation velocity of $V_{\mathrm{i}}=100 \mathrm{~km} \mathrm{~s}^{-1}$ (two times slower compared to the models in Figure 2), for the same mixing parameters as in Figure $2\left(\alpha_{\mathrm{sc}}=100\right.$, and $\alpha_{\mathrm{ov}}=0.335$ or $\left.\alpha_{\mathrm{ov}}=1.2\right)$, with the colour along the tracks showing the ratio between the stellar luminosity and the Eddington luminosity $\Gamma_{\text {Edd }}$ (computed by MESA, taking into account the gas opacity). Compared to the tracks in Figure 2, the models with $\alpha_{\mathrm{ov}}=1.2$ in Figure 3 evolve somewhat further beyond the HD limit, because of the slightly reduced rotational mixing. We point out in Figure 3 the possibility of stellar models to reside beyond the HD limit while not exceeding their Eddington luminosity. Also marked are the terminal age main sequence $\left(\mathrm{TAMS}^{2}\right)$ and final positions on the HRD ${ }^{3}$. For $\alpha_{\mathrm{ov}}=0.335$, the TAMS location is beyond the HD limit for the highest initial masses. For $\alpha_{\mathrm{ov}}=1.2$, the TAMS moves redward for the lower masses, but for the higher masses it can move bluewards. There are less pre$\mathrm{SN}$ red supergiants for $\alpha_{\mathrm{ov}}=1.2$, though for such a large extent of overshooting lower initial masses might give rise to CCSNe whose progenitors are red supergiants.

The duration of the evolutionary stage when a star is located on the HRD above the HD limit is shown in Figure 4 for various parameters. For each composition, initial mass, and overshooting scheme, there are 3 different initial rotation velocities, and we take the longest time from the three tracks. For $\alpha_{\mathrm{ov}}=0.335$, massive stars spend >100000 yr beyond the HD limit. The highest masses with MW composition do not cross the HD limit thanks to mass removal by winds (besides the models with $\alpha_{\mathrm{ov}}=0.1$ ), but as the rate depends on the metallicity, this effect is not present in the SMC and LMC models, except for those with significantly enhanced overshooting. With increasing $\alpha_{\mathrm{ov}}$, the LMC and MW models spend less time beyond the HD limit.

For the SMC the models with $30 \underset{\sim}{<} M_{\text {ZAMS }} / \mathrm{M}_{\odot} \underset{\sim}{<80}$ always cross the HD limit and spend a long time beyond it. This is because crossing the HD limit is a result of the combination of mixing and mass loss. With increased mixing, envelope material is used as fuel in the core, both increasing the core mass and luminosity and therefore the mass loss, while at the same time decreasing the envelope mass which needs to be removed by winds.

The models which use the overshooting prescription of Jermyn et al. (2018) give quantitatively similar results to those with $\alpha_{\mathrm{ov}}=1$, with $f_{\text {ov }}$ varying slightly with time and initial mass but generally close to $f_{\text {ov }} \approx 0.1$ as shown in Figure 5. Jermyn et al. (2018) motivate their prescription as describing enhanced rotational mixing caused by core anisotropy. In that sense there is a physical motivation for enhanced mixing. However, Jermyn et al. (2018) caution that their prescription appears to overestimate $f_{\text {ov }}$ relative to what Claret \&

\footnotetext{
2 The TAMS is defined as the point where the central hydrogen mass fraction drops below 0.01 .

3 The final position is marked only for models which ignited carbon in their centre, and are therefore considered to be CCSN progenitors.
} 

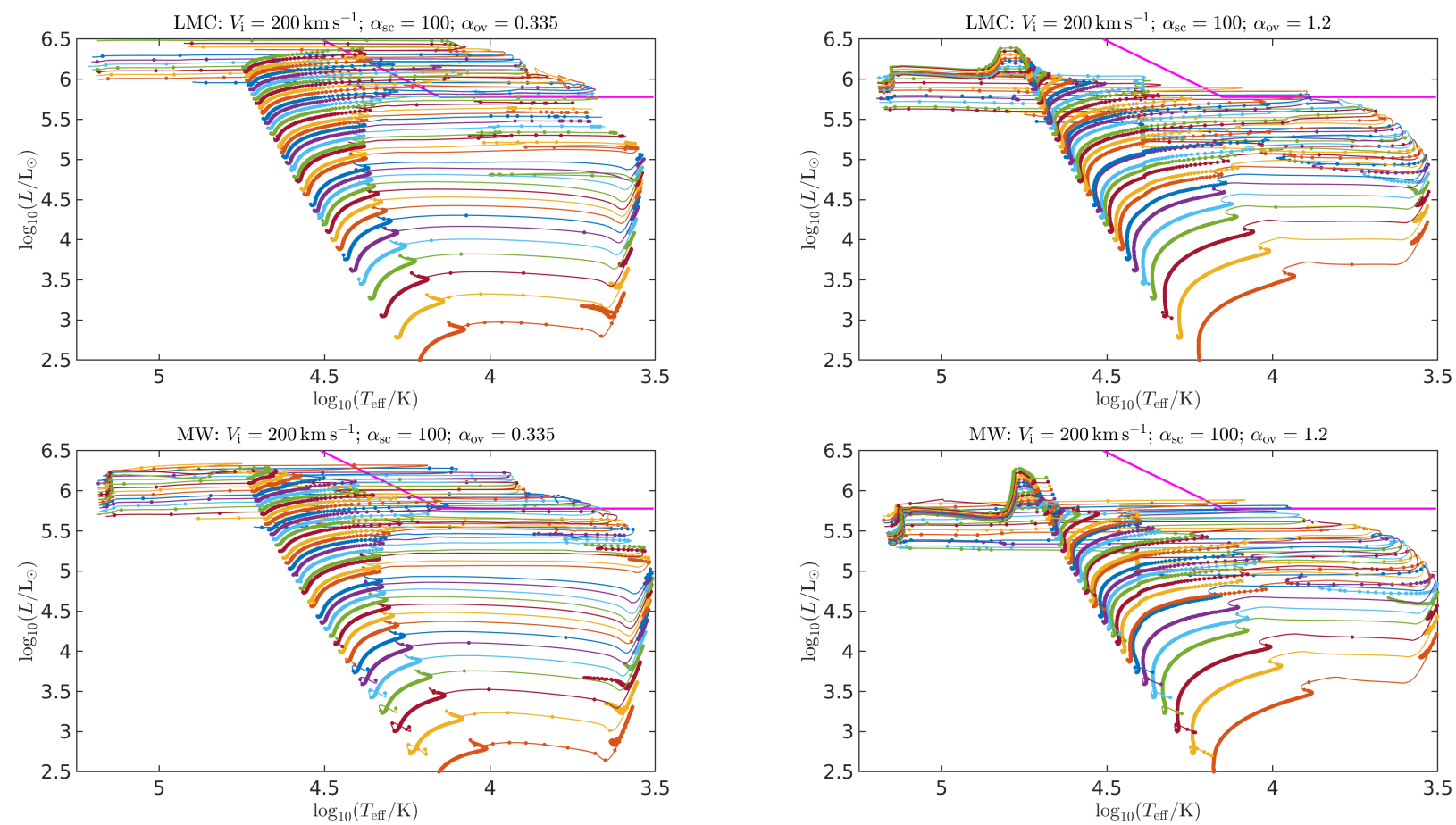

Figure 2. Hertzsprung-Russell diagrams for models with a semiconvective mixing efficiency of $\alpha_{\mathrm{sc}}=100$ and an overshoot parameter of $\alpha_{\mathrm{ov}}=0.335$ (left) and $\alpha_{\mathrm{ov}}=1.2$ (right) for LMC (top) and MW (bottom) initial compositions, with an initial rotation velocity of $200 \mathrm{~km} \mathrm{~s}^{-1}$. Initial masses are in the range $4 \mathrm{M}_{\odot} \leq M_{\mathrm{ZAMS}} \leq 107 \mathrm{M}_{\odot}$. Models every $50000 \mathrm{yr}$ are marked. The thick magenta line maps the HD limit.
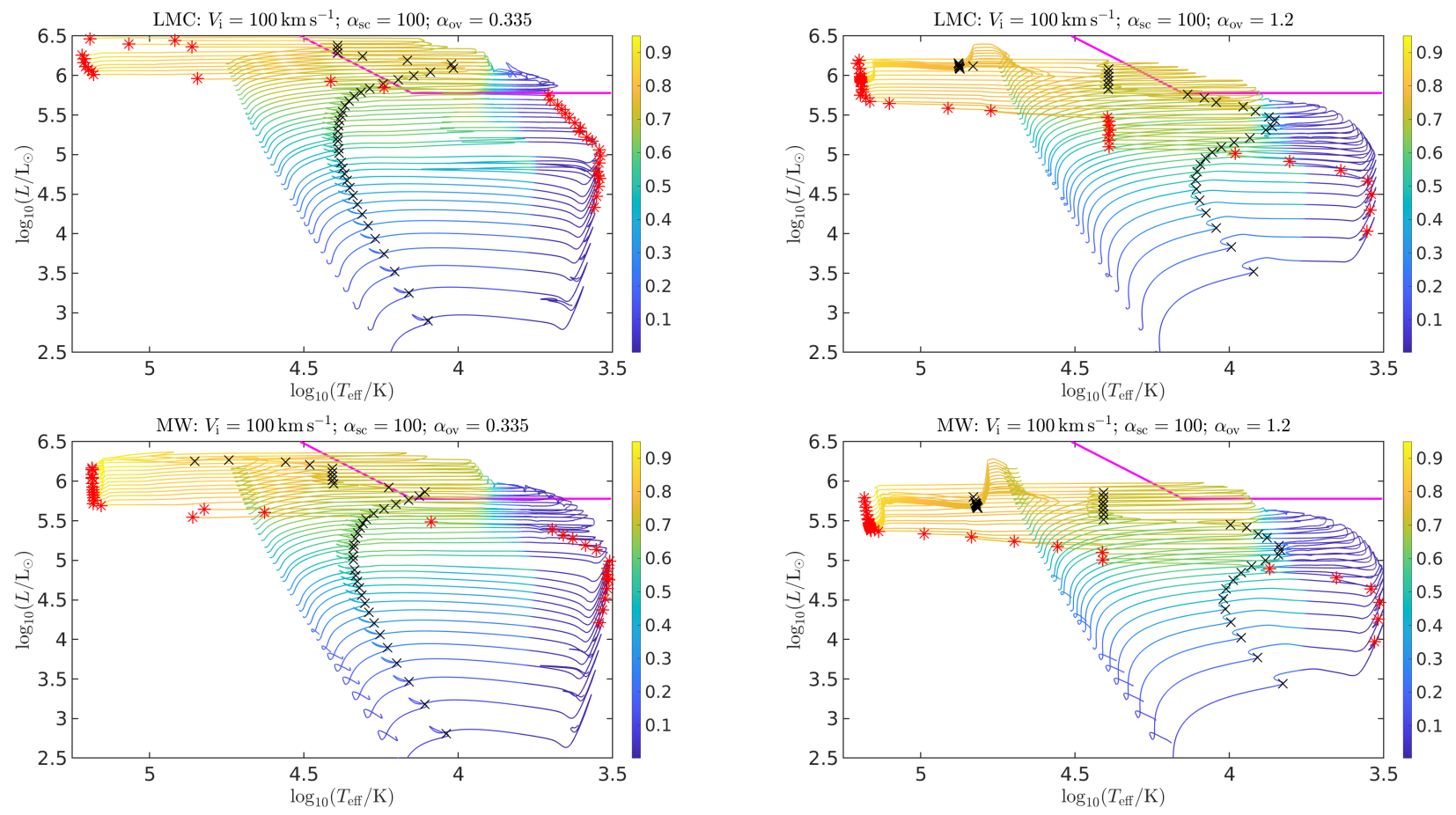

Figure 3. Hertzsprung-Russell diagrams for models with a semiconvective mixing efficiency of $\alpha_{\mathrm{sc}}=100$ and an overshoot parameter of $\alpha_{\mathrm{ov}}=0.335$ (left) and $\alpha_{\mathrm{ov}}=1.2$ (right) for LMC (top) and MW (bottom) initial compositions, with an initial rotation velocity of $100 \mathrm{~km} \mathrm{~s}^{-1}$. Initial masses are in the range $4 \mathrm{M}_{\odot} \leq M_{\text {ZAMS }} \leq 107 \mathrm{M}_{\odot}$. The line colour follows the surface Eddington factor. Black crosses show the points in the evolution where core hydrogen burning ends, and red asterisks mark the end of the evolution, when core carbon burning ends. The thick magenta line maps the HD limit. 

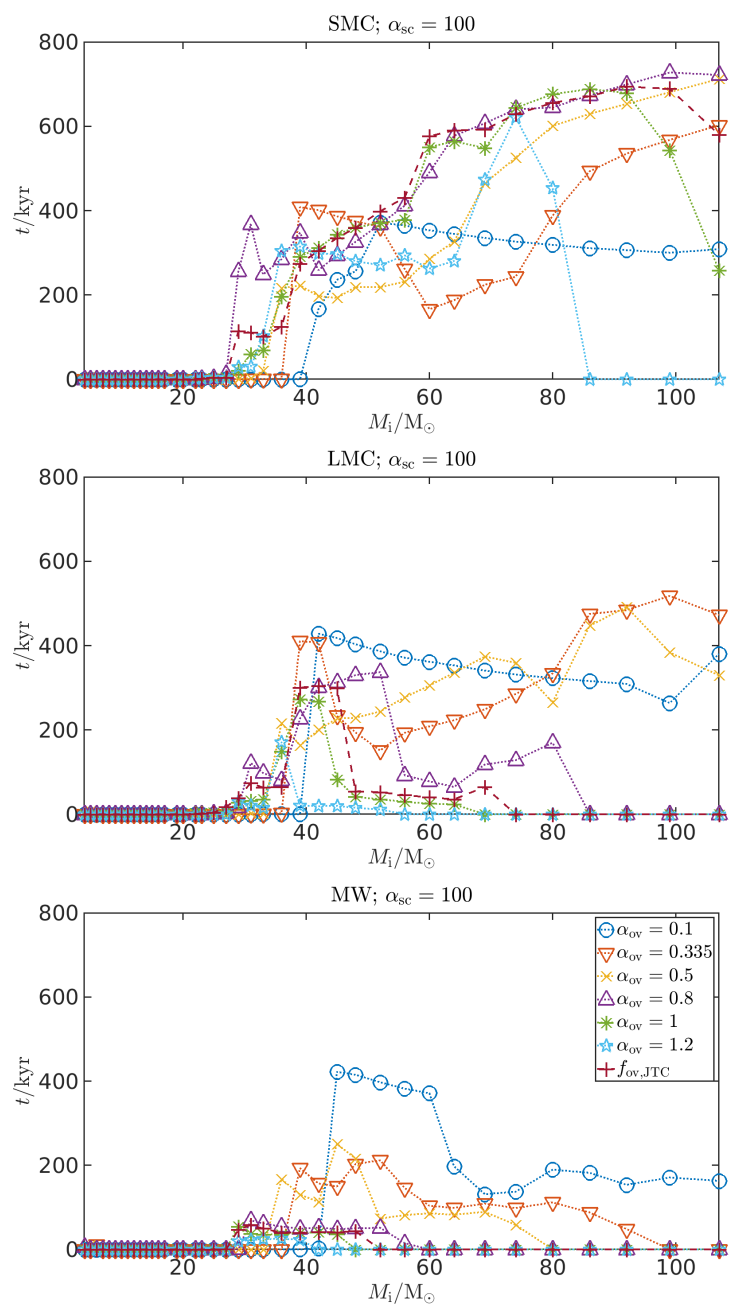

Figure 4. Time spent beyond the HD limit as function of ZAMS mass for SMC (top), LMC (middle) and MW (bottom) composition, for different core overshooting prescriptions. For each initial mass, the maximal value out of all initial rotation rates is taken. Points labeled as $f_{\text {ov,JTC }}$ use the exponential overshooting coefficient $f_{\text {ov }}$ as function of core properties described by Jermyn et al. 2018.

Torres (2017) infer from observations. Furthermore, while the usage of $f_{\text {ov }}$ is convenient, their mixing mechanism does not exhibit an exponentially decaying geometry like the $f_{\text {ov }}$ prescription assumes and so this implementation does not accurately describe the physics. Our results imply that there is a strong motivation to further investigate the mixing mechanism proposed by Jermyn et al. (2018).

\section{STATISTICAL COMPARISONS BETWEEN SYNTHETIC POPULATIONS AND OBSERVATIONS}

\subsection{Our sample compilation}

We compile the luminosities and temperatures of all known CSGs in the Magellanic Clouds with a luminosity of $\log _{10}\left(L / L_{\odot}\right)=4.7$ or higher. Unlike Davies et al. (2018), who only considered RSGs, we consider RSGs, YSGs, and cool BSGs. The reason is that the HD limit stretches over a large region on the HRD populated by these various spectral types. For simplicity, we only consider here the horizontal part of the HD limit, which extends up to effective

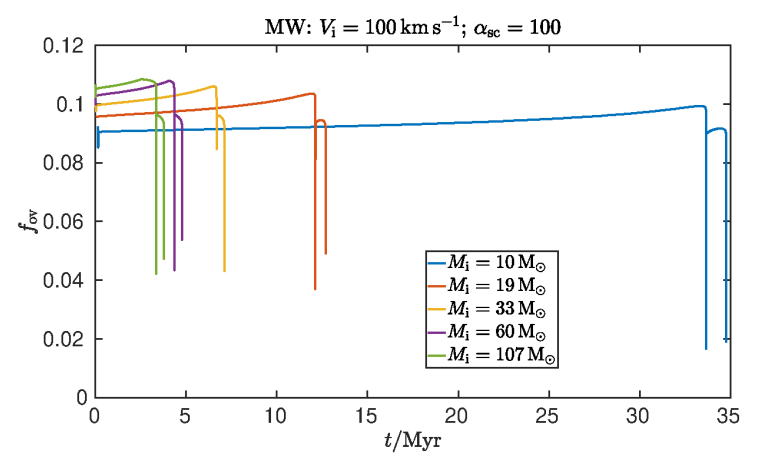

Figure 5. Exponential decay scale as fraction of the pressure scale height as function of time for several models with MW composition and an initial rotation velocity of $V_{\mathrm{i}}=100 \mathrm{~km} \mathrm{~s}^{-1}$ as calculated by using equation (1).

temperatures of $\approx 12.5 \mathrm{kK}$. Following standard convention (e.g., Drout et al. 2012), we define RSGs, YSGs, and cool BSGs in the temperature regimes $T_{\text {eff }} \leq 4800 \mathrm{~K}, 4800 \mathrm{~K}<T_{\text {eff }}<7500 \mathrm{~K}$, and $7500 \leq T_{\text {eff }} \leq 12500 \mathrm{~K}$, respectively.

For the LMC, we cross-match the RSG list of Davies et al. (2018) with the RSG-YSG list of Neugent et al. (2012). For targets that appear in both compilations, we adopt temperatures and luminosities from Davies et al. (2018), who derived these parameters from a complete spectral energy distribution (SED) fitting. When temperatures are not specified, we use calibrations between spectral types and temperatures by Tabernero et al. (2018) to derive the temperature. Since both studies claim to be complete for $\log _{10}\left(L / \mathrm{L}_{\odot}\right) \geq 4.7$, we only include objects exceeding this threshold.

The catalogue of Neugent et al. (2012) also includes stars hotter than $7500 \mathrm{~K}$, which are considered BSGs. Since their study is not necessarily complete for BSGs, we extended our catalogue by retrieving the Gaia DR2 catalogue (Gaia Collaboration 2018) centered on the LMC with a search radius of 5.5 degrees. To identify cool BSG candidates, we filtered all LMC stars in the Gaia DR2 catalogue with Gaia fluxes fulfilling the criteria $-0.1<B p-R p<$ $0.6 \mathrm{mag}$ and $G<15.5 \mathrm{mag}$, accounting for a typical reddening value of $E_{B-V}=0.09$ (e.g. Fitzpatrick \& Garmany 1990). We then crossmatched our list with the SIMBAD catalogue to retrieve spectral types using the Vizier X-MATch service. Main references are Sanduleak (1970), Ardeberg et al. (1972), Stock et al. (1976), Evans et al. (2006), and Urbaneja et al. (2017). All identified targets were classified before and have spectral types consistent with BSGs, and the majority of those were included in previous spectroscopic analyses of CSGs in the LMC.

All stars with spectral types earlier than B7 in our final list were removed, including a few WR stars. Finally We included four LBVs from the compilation given by Smith (2019).

For all remaining objects, we extracted radial velocities (RVs) and proper motions (PMs) from the SIMBAD database (Wenger et al. 2000). The sources of the RVs were predominantly the Gaia DR2 catalogue (Gaia Collaboration 2018), Massey \& Olsen (2003), Neugent et al. (2012), Fehrenbach (1972), and Fehrenbach \& Duflot (1982). PMs originate in the Gaia DR2 catalogue for all sources but ten, for which they are retrieved from Gaia DR1 (Lindegren et al.

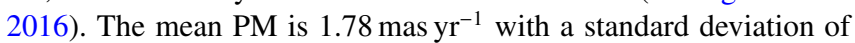
$0.3 \mathrm{mas} \mathrm{yr}^{-1}$, which reflects the measurement limit of Gaia. There

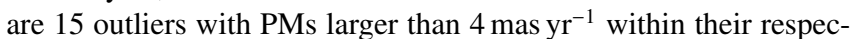
tive errors, and they are omitted from our sample to ensure that we do not include foreground Galactic objects.

When available, $T_{\text {eff }}$ and $\log L$ values for the cool BSGs were 
adopted from Urbaneja et al. (2017) and Smith (2019). Otherwise, we used spectral-type calibrations by Fitzpatrick \& Garmany (1990) to derive the effective temperatures and estimated the extinction parameters based on the expected intrinsic colours. We then used bolometric corrections following Flower (1996) and Torres (2010), assuming a distance of $49.97 \mathrm{kpc}$ (Pietrzyński et al. 2013). The final list for the LMC comprises 375 stars: 265 RSGs, 39 YSGs, and 71 cool BSGs (four of which are LBVs).

For the SMC, we repeat this procedure using the RSGs listed by Davies et al. (2018) and the YSGs listed by Neugent et al. (2010). The RVs, PMs, and spectral types are again extracted using SIMBAD. The RVs and PMs originate predominantly from the Gaia DR2 catalogue, but also from Massey \& Olsen (2003), Neugent et al. (2010), and González-Fernández et al. (2015). The spectral types are retrieved from Feast et al. (1960), Dubois et al. (1977), Humphreys (1983), Lennon (1997), and Dufton et al. (2000). Again, all objects earlier than B7 are removed. We identify about ten outliers with

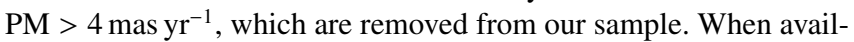
able, $T_{\text {eff }}$ and $\log L$ values for the cool BSGs were adopted from Dufton et al. (2000). Otherwise, we use calibrations by Evans \& Howarth (2003) to derive the effective temperatures, make the same assumptions as Neugent et al. (2010) regarding the reddening and the distance towards the SMC, and use the same relations as above to derive the bolometric corrections and luminosities. The final list comprises 179 stars: 140 RSGs, 7 YSGs, and 32 cool BSGs.

We note that accurate derivations of $T_{\text {eff }}$ and $\log L$ should rely on the fitting of SEDs. However, given the statistical nature of our study, the calibrations used above should be sufficient for our purpose (e.g. Neugent et al. 2010, 2012).

\subsection{Population synthesis}

We construct synthetic populations by generating random initial masses according to a Salpeter IMF. The initial rotation velocity is chosen according to the observed distributions for the LMC (Ramachandran et al. 2018b) and the SMC (Ramachandran et al. 2019). For both the initial mass and velocity, the nearest values available in our models are used to chose a stellar evolution track to follow, rather than interpolating between tracks. This results in 58\% of SMC models being assigned an initial rotation velocity of $100 \mathrm{~km} \mathrm{~s}^{-1}, 31 \%$ getting $200 \mathrm{~km} \mathrm{~s}^{-1}$ and $11 \%$ with $300 \mathrm{~km} \mathrm{~s}^{-1}$. For the LMC the corresponding percentages are $80 \%, 19 \%$ and $1 \%$.

The stellar age is chosen according to a uniform distribution, corresponding to a constant star-formation rate (SFR) ${ }^{4}$. The stellar properties are interpolated from the evolutionary track according to the generated stellar age. If the generated age is longer than the lifetime of the computed stellar evolution track, the star is discarded.

Random stars are generated until the combined number of RSGs and YSGs $\left(T_{\text {eff }}<7500 \mathrm{~K}\right)$ in the luminosity interval $4.7 \leq$ $\log _{10}\left(L / \mathrm{L}_{\odot}\right)<5.2$ matches the observed number - this is our normalisation. For the SMC this gives 134 red and yellow supergiants, and for the LMC 274. An example of a synthetic population is presented in Figure 6.

\subsection{Observations vs. simulations}

For each of the 17 sets of modelling assumptions (Table 2) we generate 25 random realisations of such populations to get an error esti-

\footnotetext{
4 This is a reasonable assumption as we are interested in young stars, though in general the SFR is not constant in the SMC and the LMC (Section 5.4).
}
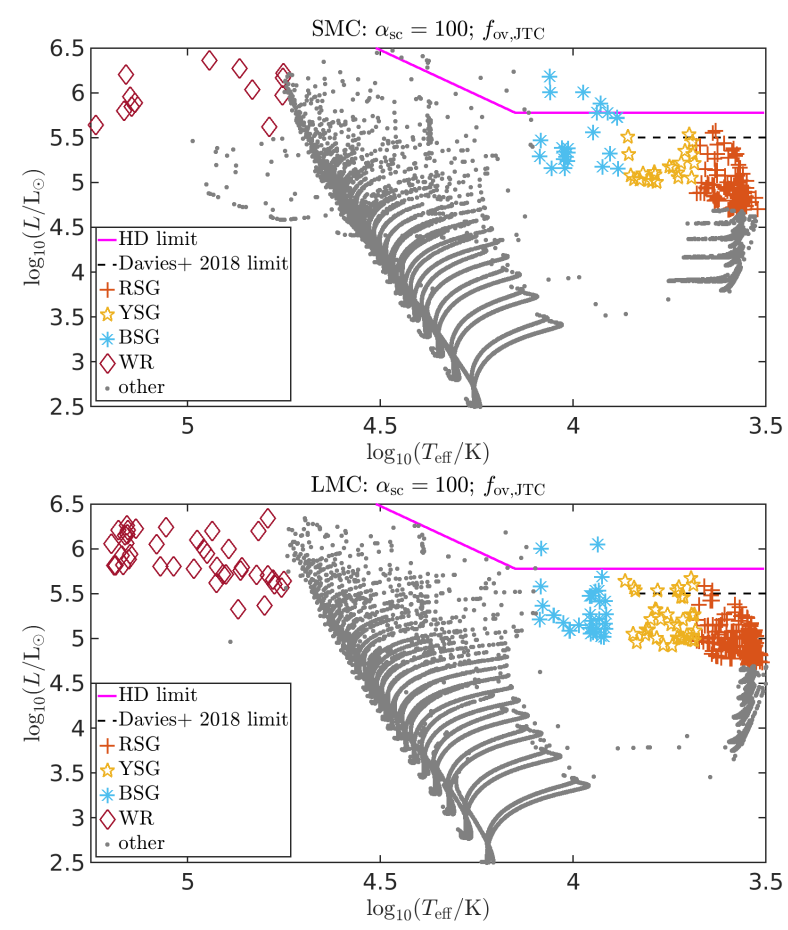

Figure 6. Example synthetic populations of stars with SMC (top) and LMC (bottom) initial compositions.

mate for the computed numbers. We count the number of stars generated which are over-luminous, i.e. those with $\log _{10}\left(L / \mathrm{L}_{\odot}\right) \geq 5.4$. The results for the 850 synthetic populations are summarised in Table 3 and Table 4, and in Figure 7 we show the number of overluminous supergiants in our synthetic populations for all models which employ step overshooting. Tables 3 and 4 show that looking only at red supergiants leads to underestimating the excess of over-luminous stellar models. The excess generally decreases with increasing $\alpha_{\mathrm{ov}}$, although for efficient semiconvective mixing $\left(\alpha_{\mathrm{sc}}=\right.$ $100)$ the behaviour is non-monotonic for the red and yellow regimes. The models employing the overshooting prescription given by equation (1) produce very similar results to those with $\alpha_{\mathrm{ov}}=1$.

In Figure 8 we show the simulated luminosity distribution for a few sets of mixing parameters compared to the observed distribution. For the RSGs, the simulated distribution is similar to the observed distribution for most mixing assumptions, with a small excess in the simulations for some cases, such as the combination of $\alpha_{\mathrm{ov}}=0.335$ and $\alpha_{\mathrm{sc}}=1$. The excess in the simulations increases when including also the YSGs, especially as there is a non-negligible number of such stars in the simulated populations, while the number of observed luminous YSGs is small. When including also cool BSGs, i.e. all stars with $T_{\text {eff }} \leq 12500 \mathrm{~K}$, the excess of over-luminous stars in the simulated populations becomes even larger. An excess in the lower luminosities for BSGs is acceptable as we do not expect the sample to be complete for these temperatures, but higher luminosity stars $\left(\log _{10}\left(L / \mathrm{L}_{\odot}\right) \geq 5.4\right)$ would definitely be observed, and therefore the simulations cannot be taken to properly account for the stellar population.

In Figure 9 we show the ratio between the number of RSGs with $4.7 \leq \log _{10}\left(L / \mathrm{L}_{\odot}\right)<5.4$ to the number of YSGs in the same luminosity range. This ratio is a good test for mixing in evolved massive stars. Schootemeijer et al. (2019) discuss a similar ratio, but between the numbers of RSGs and BSGs. We note also that the expansion and colour of supergiants depends on the treatment of convection, 

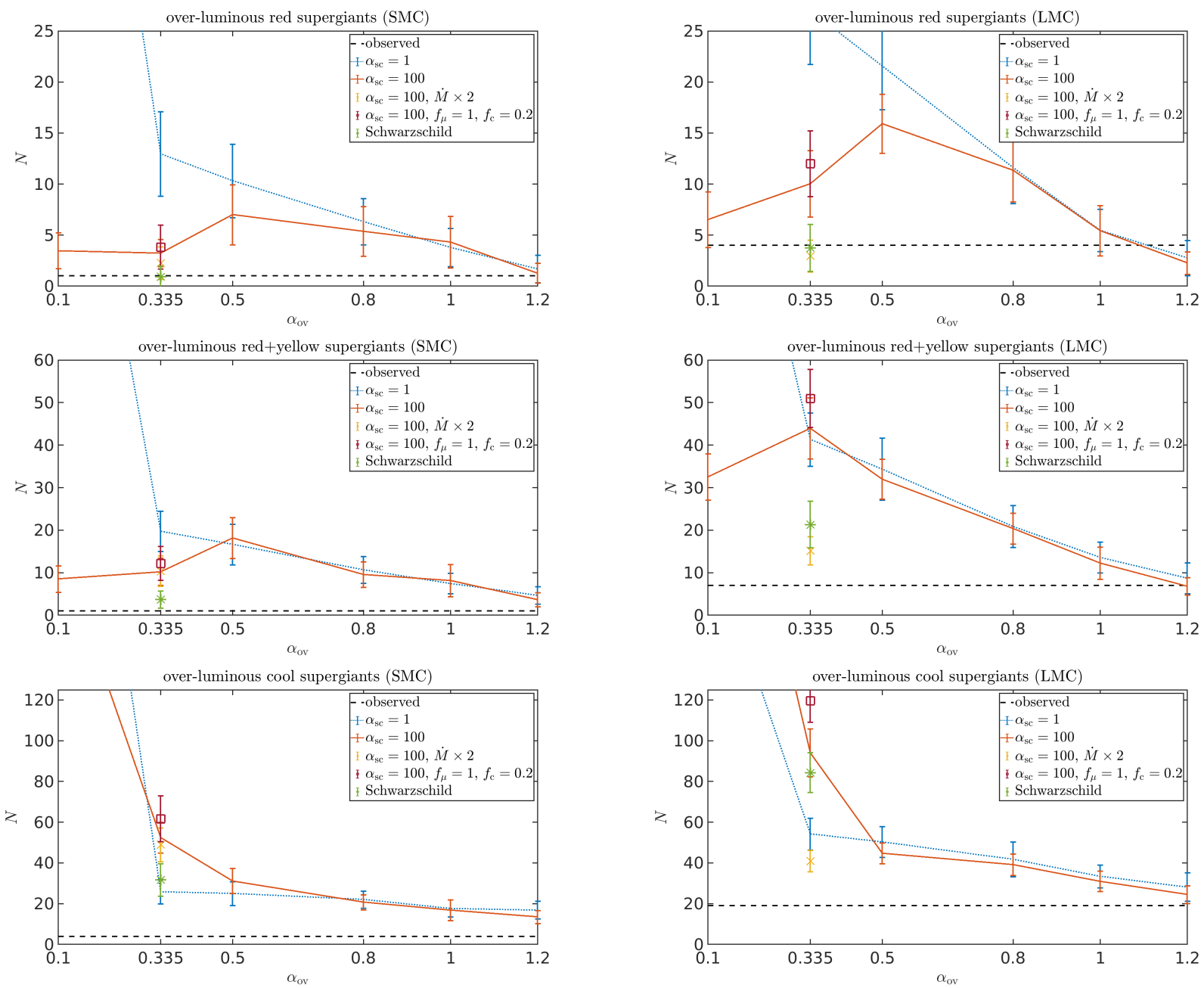

Figure 7. Number of supergiant stars with $\log _{10}\left(L / \mathrm{L}_{\odot}\right) \geq 5.4$ in synthetic populations as function of the overshooting extent, for two values of the semiconvective mixing efficiency, as well as models employing the Schwarzschild stability criterion instead of Ledoux, for the SMC (left) and the LMC (right). For $\alpha_{\mathrm{sc}}=100$, models with doubled mass loss or alternative parameters for rotational mixing are also shown. The top panels show the number of over-luminous red $\left(T_{\text {eff }} \leq 4800 \mathrm{~K}\right)$ supergiant stars, the middle panels show red and yellow $\left(T_{\text {eff }}<7500 \mathrm{~K}\right)$ supergiant stars, and the bottom panels show cool $\left(T_{\text {eff }} \leq 12500 \mathrm{~K}\right)$ supergiant stars.

and employing MLT++ results in very luminous stars being hotter (Klencki et al. 2020). In the present study we focus on the most luminous stars, whose numbers are small compared to those with lower luminosities which mostly affect the YSG/RSG (or BSG/RSG) ratio. Supergiants of all colours need to be taken into account, to separate the issue of the number ratio of different colours from the issue of the HD limit.

\section{SOURCES OF UNCERTAINTY AND THEIR IMPACT}

\subsection{Mass loss}

Alongside mixing, continuous or eruptive mass loss is a key parameter that is responsible for stripping a star of its H-rich envelope. The larger the mass loss, the less likely the star is to cross the HD limit. Motivated by the apparent $Z$-independence of the HD limit, we chose to focus on mixing here. However, to shed more light on the interplay between mass loss and mixing in this context, we also provide a set of models with mass-loss rates that are boosted by a factor of two. Recent theoretical and empirical determinations of mass-loss rates during the $\mathrm{OB}$ and the RSG phases (e.g., Ramachandran et al. 2019; Björklund et al. 2020; Beasor et al. 2020) rather suggest that standard prescriptions such as those used here already lead to an overestimation of the mass loss. Nevertheless, considering the poor understanding of eruptive mass loss, we explore the impact of an increased mass loss. We do this for the case of $\alpha_{\mathrm{ov}}=0.335$ and $\alpha_{\text {sc }}=100$.

The impact of boosting the mass loss throughout the stellar evolution by a factor of two is shown in Figure 7. As could be anticipated, boosting the mass loss helps reduce the excess of CSGs above the HD limit. However, even with increased mass loss, the discrepancy remains significant. Moreover, while it leads to an appreciable improvement in the case of the LMC, the improvement is negligible in the SMC, which again opposes the apparent $Z$-independence of the HD limit. To conclude, while continuous mass loss will play a role in shaping the HD limit, there is little support that it alone can explain the observations. It is well possible that a $Z$-independent rapid phase of mass loss (e.g., in a CSG or LBV phase) needs to be invoked to avoid the discrepancy between observations and theory, but a consistent physical framework for implementing it is still missing. 

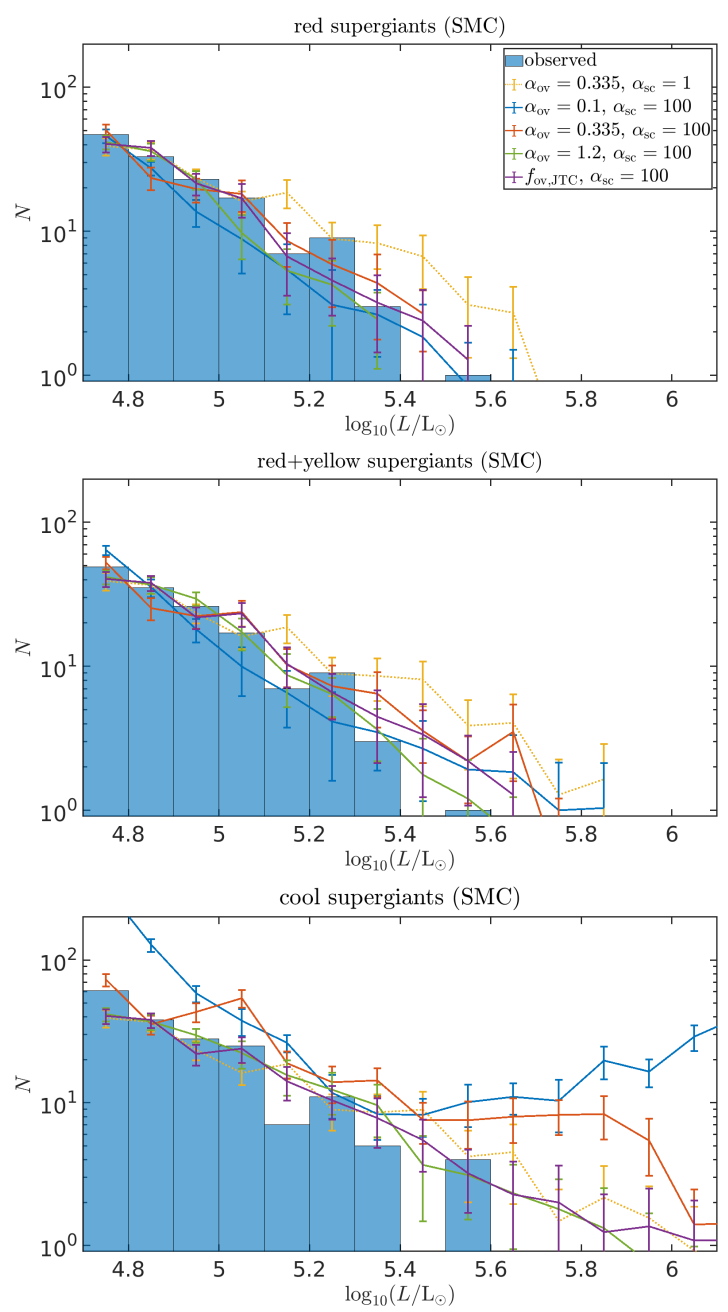
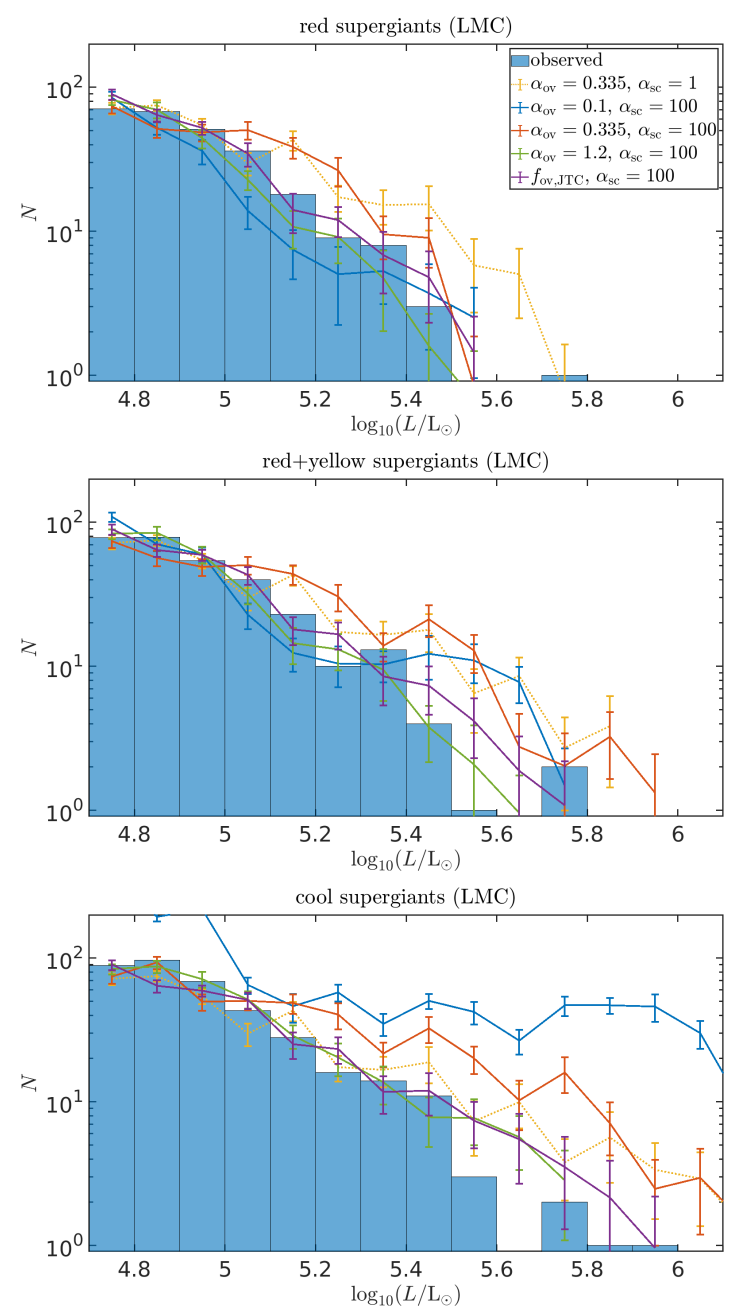

Figure 8. Number of supergiant stars as function of luminosity, for a sample of five different sets of mixing parameters compared to the observed distributions for the SMC (left) and the LMC (right). The top panels show the number of RSGs ( $T_{\text {eff }} \leq 4800 \mathrm{~K}$ ), the middle panels show RSGs and YSGs ( $T_{\text {eff }}<7500 \mathrm{~K}$ ), and the bottom panels show cool $\left(T_{\mathrm{eff}} \leq 12500 \mathrm{~K}\right)$ supergiant stars.

\subsection{Mixing prescriptions}

The main uncertainty in stellar evolution modelling on which we focus in this work is mixing in stellar interiors. Therefore, in addition to the efficiency of semiconvection and the extent of overshooting, the effects of a couple of other mixing assumptions were tested. While we mostly used the Ledoux criterion for defining the convective boundary, in one set of models (with $\alpha_{\mathrm{ov}}=0.335$ ) we used the Schwarzschild criterion instead. The stability criterion employed affects not only the boundary of the convective core, but also intermediate regions between the core boundary and the stellar photosphere and the position of models in the HRD (Georgy, Saio \& Meynet 2014). The analysis of our synthetic populations generated from stellar evolution tracks which employed the Schwarzschild criterion yields a somewhat reduced excess of over-luminous CSGs compared to the analysis with the Ledoux criterion and the same overshooting extent. Similarly to our results with boosted mass loss (Section 5.1), the excess remains non-negligible, especially when considering the entire relevant temperature range.

Up until now we have discussed mixing only for convective regions and near their boundaries. In models of rotating stars, considerable mixing occurs also in radiative regions, owing to various instabilities (e.g., Heger et al. 2000). In one set of models we changed the rotational mixing parameters in radiative regions to $f_{c}=0.2$ and $f_{\mu}=1$, as described in Section 2.3. Our analysis of the synthetic populations generated from tracks with these alternative mixing parameters yields almost no change in the CSG excess (Fig. 7). This might be a result of the possible degeneracy of these parameters (Chieffi \& Limongi 2013).

As an additional test of the sensitivity to the implementation of rotational mixing, we also compare our results to the Geneva tracks with MW and SMC compositions (Ekström et al. 2012; Georgy et al. 2013). In Figure 10 we show the time spent beyond the HD limit for several of our modelling assumptions and for the Geneva models, for which rotational mixing is treated as an advective-diffusive process, compared to the diffusion approximation adopted by MESA. The Geneva models spend time periods beyond the HD limit rather similar to our tracks computed with MESA. While a quantitative comparison using synthetic populations will give a more definitive answer, we surmise that the computed excess of CSGs will be similar with the Geneva tracks. 
Table 3. Excess of over-luminous stars in synthetic populations.

\begin{tabular}{lcccc}
\hline \hline & overshooting & $\alpha_{\text {sc }}$ & $N_{\text {excess,RSG }+ \text { YSG }}$ & $N_{\text {excess,RSG }}$ \\
\hline SMC & $\alpha_{\text {ov }}=0.1$ & 1 & $152 \pm 15$ & $79 \pm 10$ \\
SMC & $\alpha_{\text {ov }}=0.335$ & 1 & $20 \pm 5$ & $13 \pm 4$ \\
SMC & $\alpha_{\text {ov }}=0.5$ & 1 & $17 \pm 5$ & $10 \pm 4$ \\
SMC & $\alpha_{\text {ov }}=0.8$ & 1 & $11 \pm 3$ & $6 \pm 2$ \\
SMC & $\alpha_{\text {ov }}=1.0$ & 1 & $7 \pm 2$ & $4 \pm 2$ \\
SMC & $\alpha_{\text {ov }}=1.2$ & 1 & $5 \pm 2$ & $2 \pm 1$ \\
SMC & $f_{\text {ov }, J T C}$ & 1 & $8 \pm 3$ & $4 \pm 3$ \\
\hline SMC & $\alpha_{\text {ov }}=0.1$ & 100 & $9 \pm 3$ & $3 \pm 2$ \\
SMC & $\alpha_{\text {ov }}=0.335$ & 100 & $10 \pm 3$ & $3 \pm 1$ \\
SMC & $\alpha_{\text {ov }}=0.5$ & 100 & $18 \pm 5$ & $7 \pm 3$ \\
SMC & $\alpha_{\text {ov }}=0.8$ & 100 & $10 \pm 3$ & $5 \pm 2$ \\
SMC & $\alpha_{\text {ov }}=1.0$ & 100 & $8 \pm 4$ & $4 \pm 3$ \\
SMC & $\alpha_{\text {ov }}=1.2$ & 100 & $4 \pm 2$ & $1 \pm 1$ \\
SMC & $f_{\text {ov }, J T C}$ & 100 & $7 \pm 2$ & $4 \pm 2$ \\
\hline LMC & $\alpha_{\text {ov }}=0.1$ & 1 & $145 \pm 15$ & $103 \pm 12$ \\
LMC & $\alpha_{\text {ov }}=0.335$ & 1 & $41 \pm 6$ & $27 \pm 5$ \\
LMC & $\alpha_{\text {ov }}=0.5$ & 1 & $34 \pm 7$ & $22 \pm 4$ \\
LMC & $\alpha_{\text {ov }}=0.8$ & 1 & $21 \pm 5$ & $12 \pm 4$ \\
LMC & $\alpha_{\text {ov }}=1.0$ & 1 & $14 \pm 4$ & $5 \pm 2$ \\
LMC & $\alpha_{\text {ov }}=1.2$ & 1 & $9 \pm 4$ & $3 \pm 2$ \\
LMC & $f_{\text {ov }, J T C}$ & 1 & $14 \pm 3$ & $6 \pm 3$ \\
\hline LMC & $\alpha_{\text {ov }}=0.1$ & 100 & $33 \pm 5$ & $7 \pm 3$ \\
LMC & $\alpha_{\text {ov }}=0.335$ & 100 & $44 \pm 7$ & $10 \pm 3$ \\
LMC & $\alpha_{\text {ov }}=0.5$ & 100 & $32 \pm 5$ & $16 \pm 3$ \\
LMC & $\alpha_{\text {ov }}=0.8$ & 100 & $20 \pm 4$ & $11 \pm 3$ \\
LMC & $\alpha_{\text {ov }}=1.0$ & 100 & $12 \pm 4$ & $5 \pm 2$ \\
LMC & $\alpha_{\text {ov }}=1.2$ & 100 & $7 \pm 2$ & $2 \pm 1$ \\
LMC & $f_{\text {ov }, J T C}$ & 100 & $14 \pm 3$ & $7 \pm 3$ \\
\hline & & & & \\
\hline
\end{tabular}

Table 4. Excess of over-luminous stars in additional synthetic populations.

\begin{tabular}{lccc}
\hline \hline & model assumptions & $N_{\text {excess,RSG+YSG }}$ & $N_{\text {excess,RSG }}$ \\
\hline SMC & Schwarzschild & $4 \pm 2$ & $1 \pm 1$ \\
SMC & $f_{c}=0.2, f_{\mu}=1$ & $12 \pm 4$ & $4 \pm 2$ \\
SMC & $\eta_{\mathrm{w}}=2$ & $10 \pm 4$ & $2 \pm 2$ \\
\hline LMC & Schwarzschild & $21 \pm 5$ & $4 \pm 2$ \\
LMC & $f_{c}=0.2, f_{\mu}=1$ & $51 \pm 7$ & $12 \pm 3$ \\
LMC & $\eta_{\mathrm{w}}=2$ & $15 \pm 3$ & $3 \pm 2$ \\
\hline
\end{tabular}

\subsection{Multiplicity}

In principle, the presence of a close companion (orbital period $P \lesssim 3 \mathrm{yr}$ ) would inhibit the expansion of its companion. Because a large fraction of massive stars interact with a companion during their evolution (Sana et al. 2012; Moe \& Di Stefano 2017), the effects of binaries on the simulated populations of CSGs and the HD limit should be taken into account. Given the many uncertain parameters that describe the properties and evolution of binaries, we decided to not account for multiplicity here. One may naively expect that binary interactions may be highly relevant for explaining the HD limit, since they can prevent stars from becoming CSGs. However, because the total number of RSGs and YSGs is used for the normalisation of our synthetic populations, and because it itself will be affected by binary interactions, the effect of stellar multiplicity is not trivial. If the binary separation shrinks as the binary mass increases, then one may expect binary interactions to help resolve
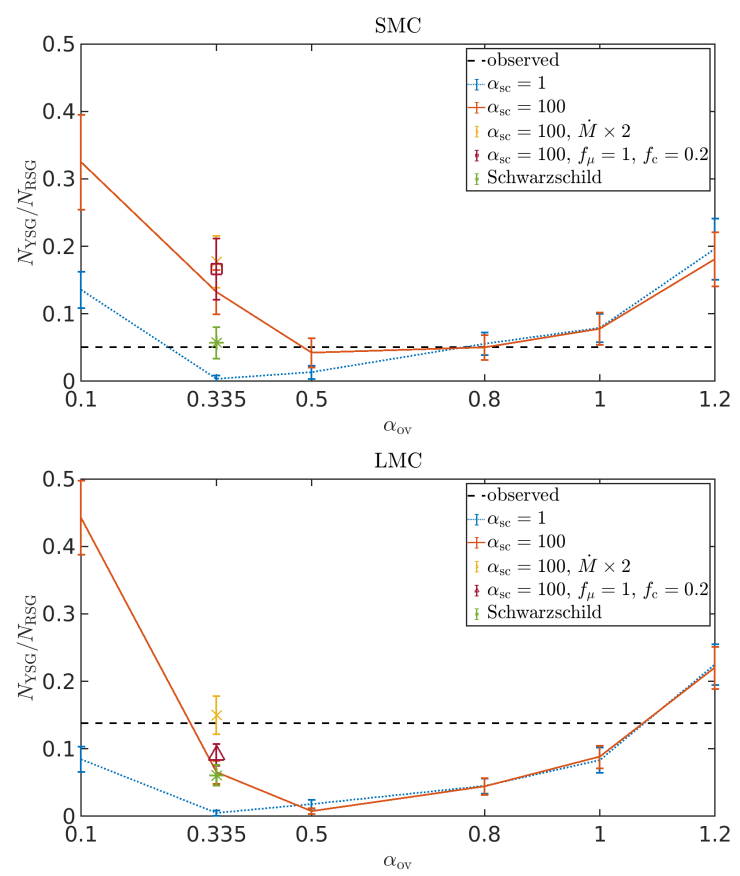

Figure 9. Number of YSG stars relative to the number of RSG stars in synthetic populations as function of the overshooting extent, for two values of the semiconvective mixing efficiency, for the SMC (top) and the LMC (bottom).

the discrepancy. However, solid evidence for this at the upper-mass end is currently lacking. Addressing this question in future studies should be helpful to quantitatively constrain the impact of multiplicity on the HD limit.

\subsection{Star-formation history}

There are strong indications that the recent star-formation history in the Magellanic Clouds, especially in the SMC, was not continuous but is rather characterised by various peaks over the past $100 \mathrm{Myr}$ (Indu \& Subramaniam 2011; Ramachandran et al. 2019; Fulmer et al. 2020). As such, this can have an important impact on the synthetic populations obtained, and hence on the final conclusions. However, it is unlikely that star-formation history alone can explain the discrepancy in the SMC and the LMC simultaneously. Moreover, the presence of WR stars in both galaxies that span a substantial luminosity range (Hainich et al. 2014, 2015; Shenar et al. 2016, 2019) suggests that a similar distribution of CSGs across the luminosity range could be anticipated. Given the many uncertainties, repeating this investigation with detailed star-formation histories is beyond the scope of our work, but should be explored in future studies.

\section{SUMMARY AND DISCUSSION}

We evolve numerous grids of stellar evolution tracks, for MW, LMC and SMC compositions, with a variety of mixing prescriptions. We find that enhanced mixing diverts stellar evolution tracks from the "forbidden region" defined by the HD limit (Figs. 2-3). Based upon these grids of stellar models we construct synthetic populations with initial compositions appropriate for the LMC and SMC, and make quantitative comparisons with the observed populations of $\operatorname{cool}\left(T_{\text {eff }} \leq 12.5 \mathrm{kK}\right)$ supergiant stars in these galaxies (Section 4). 

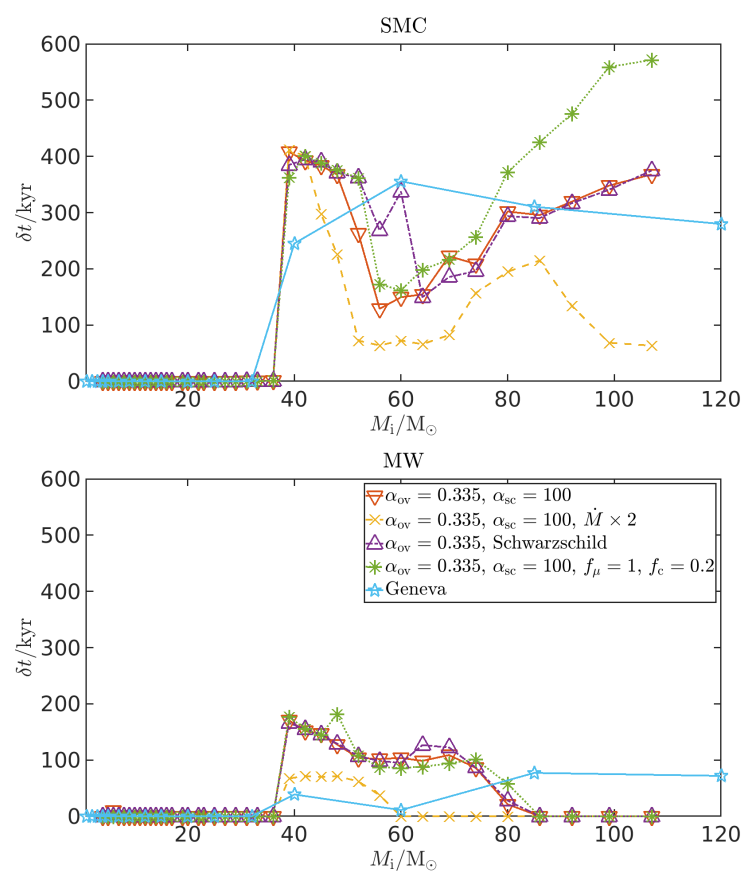

Figure 10. Time spent beyond the HD limit as function of ZAMS mass for SMC (top) and MW (bottom) composition, for stellar evolution tracks as described in the inset.

We find that enhanced mixing reduces the excess of over-luminous CSGs in our simulated stellar populations. While for RSGs there does not seem to be a severe problem, the tension between observations and simulations increases with increasing the upper temperature cutoff. We can therefore consider the existence of a "Cool Supergiant Problem" as the apparent mismatch between observed supergiants and the results of stellar evolution models.

When considering only RSGs, we find that the dependence of the excess on the overshooting extent is non-monotonic for efficient semiconvective mixing $\left(\alpha_{\mathrm{sc}}=100\right)$. Recent studies by Schootemeijer et al. (2019) and Higgins \& Vink (2020) advocate for highly efficient mixing in regions of semiconvection to account for the properties of supergiants in the LMC and SMC. Schootemeijer et al. (2019) also claim that convective overshooting can be constrained by the properties of the populations, such as the ratio between RSGs and BSGs. We differ from Schootemeijer et al. (2019) in two regards: (i) We consider much higher values of $\alpha_{\mathrm{ov}}$, beyond an apparent extremum around $\alpha_{\mathrm{ov}} \approx 0.5$; (ii) Schootemeijer et al. (2019) focus on the bulk of the supergiant population, while we investigate the stars with the highest luminosities, which are a small fraction of the overall population.

Higgins \& Vink (2020) suggest that the HD limit can be explained with decreased overshoot mixing, $\alpha_{\mathrm{ov}}=0.1$, though they consider only the red part of the evolution. We show that for red supergiants taking $\alpha_{\mathrm{ov}}=0.1$ together with $\alpha_{\mathrm{sc}}=100$ indeed gives a reasonable account of the supergiant populations in the LMC and SMC (Figs. 7-8). This is explained by the significant effect of efficient semiconvection in increasing the effective surface temperatures of the stellar models. Thus, for efficient semiconvection, core He-burning stars tend to appear as YSGs or BSGs instead of RSGs. However, this does not solve the supergiant excess beyond the HD limit, but merely "sweeps it under the carpet". When we consider the entire temperature range to which the horizontal HD limit applies $\left(T_{\text {eff }} \lesssim 12.5 \mathrm{kK}\right.$ ) we find that there is a large excess of stellar models in our simu- lated populations. As Higgins \& Vink (2020) did not consider the same temperature range as us, there is no discrepancy between the results. It is important to realise that the HD limit is not limited only to RSGs, and hence all relevant temperatures need to be included.

It is also evident from our results that the extent of overshooting affects the formation of WR stars. Enhanced mixing reduces the mass threshold for a star to remove its envelope through winds, and therefore affects the necessity of binary interactions in forming WR stars (Shenar et al. 2020), as well as decreasing the single-star evolution WR luminosity threshold. For example, Figs. 2-3 show that increasing $\alpha_{\text {ov }}$ from 0.335 to 1.2 reduces the lowest luminosity of a WR star formed through single-star evolution from $\log _{10}\left(L / \mathrm{L}_{\odot}\right) \approx 6$ to $\approx 5.5$ for the $\mathrm{LMC}$, and from $\approx 5.5$ to $\approx 5.25$ for the MW.

Our results also have implications for energetic transient astrophysical phenomena. Firstly, the initial mass threshold for corecollapse supernovae is lower for enhanced mixing. Secondly, the position in the HRD where stellar models end their lives is substantially hotter for enhanced mixing. This has implications for the so-called "Red Supergiant Problem", which is the apparent discrepancy between the most luminous progenitor of a Type IIP supernova and the most luminous known RSGs (Smartt et al. 2009; Horiuchi et al. 2014; Davies \& Beasor 2018, 2020; Kochanek 2020). Our models with lower overshooting values exhibit such a problem, though for enhanced mixing the more massive stars do not die as RSGs, in accordance with observations.

The formation of black hole binaries (which are progenitors of gravitational wave events) depends on the expansion of massive stars and their interaction with a companion. Klencki et al. (2020) discuss the role of metallicity in stellar expansion and interaction, as with lower metallicity stars are generally more compact because of the lower gas opacity. Smaller stellar radii imply that a smaller fraction of stars will experience significant binary interactions, such as common envelope evolution, that lead to short-period binary black holes which will merge quickly enough to produce observable gravitational wave events. Our models with enhanced mixing expand to smaller radii in general, therefore affecting the evolution towards merging black holes. Since a large fraction of massive stars interact with a companion during their evolution

Klencki et al. (2020) also discuss the role of MLT++ in relation to the HD limit. According to Klencki et al. (2020), the more limited expansion of massive stellar models employing MLT++ is favorable in terms of explaining the HD limit, and it might be more accurate for rather massive $\left(M>50 \mathrm{M}_{\odot}\right)$ stars. We note that even though we use the favorable MLT++ prescription we still find an excess of overluminous stars in the temperature range relevant for the HD limit.

It is important to stress that our study focuses on the upper-mass end. For example, applying high $\alpha_{\mathrm{ov}}$ values as invoked here can suppress blue loops (Stothers \& Chin 1991; Walmswell, Tout \& Eldridge 2015) on the HRD (Fig. 2) and prevent lower-mass stars from becoming Cepheids (Anderson et al. 2014, 2016), in contrast to observations. However, studies of intermediate-mass stars suggest a mass-dependent convective overshoot extent (Claret \& Torres 2016, $2017,2018,2019$ ). These studies suggest an increase up to $\alpha_{\mathrm{ov}} \approx 0.2$ at $M \approx 2 \mathrm{M}_{\odot}$, plateauing afterwards. A similar trend is explained by Jermyn et al. (2018). Overshooting is less well-constrained for the higher masses which are relevant for our study and for the existence of the HD limit. Models of massive stars make various calibrations for the overshooting extent, with results ranging from $\alpha_{\mathrm{ov}}=0.1$ calibrated for the mass range of 1.3-9 $\mathrm{M}_{\odot}$ (Ekström et al. 2012) to $\alpha_{\mathrm{ov}}=0.5$ for higher masses around $M>30 \mathrm{M}_{\odot}$ (Higgins \& Vink 2019). So while very high overshoot values cannot be applied to the lowest masses in our grids $\left(M=4 \mathrm{M}_{\odot}\right)$, higher mass stars might 
exhibit behaviour appropriate for enhanced mixing, with few real constraints on the extent.

In conclusion, we propose that internal mixing in massive stars might play an important part in explaining the empiric HD limit. Enhanced mixing prevents the redward evolution of stellar models towards or beyond the HD limit. We do not suggest to adopt a higher overshooting parameter, but rather that our results hint at a deficiency in the modelling of mixing in stellar interiors (e.g., Aerts et al. 2019; Schootemeijer et al. 2019). The extent of core overshooting might be highly mass-dependent, or rotational mixing is more efficient for slowly rotating stars. Moreover, it is well possible that the final explanation relies on a multitude of mechanisms (e.g., mixing, mass loss, multiplicity, star formation history; see Sect. 5). We therefore encourage future investigations of this problem that address these various mechanisms. For now, internal mixing in massive stars remains an unresolved issue in stellar modelling with broad implications, and the origin of the Humphreys-Davidson limit remains uncertain.

\section{ACKNOWLEDGMENTS}

We acknowledge the constructive report by our referee Dr. Cyril Georgy. This research has made use of the SIMBAD database and cross-match service operated at CDS, Strasbourg, France. The research has received funding from the European Research Council (ERC) under the European Union's Horizon 2020 research and innovation programme with grant number 772225: MULTIPLES. AG and TS would like to thank Danny Lennon and Ben Davies for helpful input and exchanges. The Flatiron Institute is supported by the Simons Foundation. IA is a CIFAR Azrieli Global Scholar in the Gravity and the Extreme Universe Program and acknowledges support from that program, from the European Research Council (ERC) under the European Union's Horizon 2020 research and innovation program (grant agreement number 852097), from the Israel Science Foundation (grant numbers 2108/18 and 2752/19), from the United States - Israel Binational Science Foundation (BSF), and from the Israeli Council for Higher Education Alon Fellowship.

\section{DATA AVAILABILITY STATEMENT}

The data underlying this article will be shared on reasonable request to the corresponding author.

\section{REFERENCES}

Aerts C., Mathis S., Rogers T. M., 2019, ARA\&A, 57, 35

Alastuey A., Jancovici B., 1978, ApJ, 226, 1034

Almeida L. A., et al., 2017, A\&A, 598, A84

Anderson R. I., Ekström S., Georgy C., Meynet G., Mowlavi N., Eyer L., 2014, A\&A, 564, A100

Anderson R. I., Saio H., Ekström S., Georgy C., Meynet G., 2016, A\&A, 591, A8

Ardeberg A., Brunet J. P., Maurice E., Prevot L., 1972, A\&AS, 6, 249

Beasor E. R., Davies B., Smith N., van Loon J. T., Gehrz R. D., Figer D. F., 2020, MNRAS, 492, 5994

Bestenlehner J. M., et al., 2011, A\&A, 530, L14

Björklund R., Sundqvist J. O., Puls J., Najarro F., 2020, arXiv e-prints, p. arXiv:2008.06066

Bouret J. C., Lanz T., Martins F., Marcolino W. L. F., Hillier D. J., Depagne E., Hubeny I., 2013, A\&A, 555, A1

Brott I., et al., 2011, A\&A, 530, A115
Buchler J. R., Yueh W. R., 1976, ApJ, 210, 440

Cassisi S., Potekhin A. Y., Pietrinferni A., Catelan M., Salaris M., 2007, ApJ, 661,1094

Castro N., Oey M. S., Fossati L., Langer N., 2018, ApJ, 868, 57

Chieffi A., Limongi M., 2013, ApJ, 764, 21

Claret A., Torres G., 2016, A\&A, 592, A15

Claret A., Torres G., 2017, ApJ, 849, 18

Claret A., Torres G., 2018, ApJ, 859, 100

Claret A., Torres G., 2019, ApJ, 876, 134

Crowther P. A., Dessart L., Hillier D. J., Abbott J. B., Fullerton A. W., 2002, A\&A, 392, 653

Crowther P. A., Schnurr O., Hirschi R., Yusof N., Parker R. J., Goodwin S. P., Kassim H. A., 2010, MNRAS, 408, 731

Cyburt R. H., et al., 2010, ApJS, 189, 240

Davies B., Beasor E. R., 2018, MNRAS, 474, 2116

Davies B., Beasor E. R., 2020, MNRAS, 493, 468

Davies B., Crowther P. A., Beasor E. R., 2018, MNRAS, 478, 3138

Dewitt H. E., Graboske H. C., Cooper M. S., 1973, ApJ, 181, 439

Drout M. R., Massey P., Meynet G., 2012, ApJ, 750, 97

Dubois P., Jaschek M., Jaschek C., 1977, A\&A, 60, 205

Dufton P. L., McErlean N. D., Lennon D. J., Ryans R. S. I., 2000, A\&A, 353, 311

Dufton P. L., Evans C. J., Hunter I., Lennon D. J., Schneider F. R. N., 2019, A\&A, 626, A50

Ekström S., et al., 2012, A\&A, 537, A146

Evans C. J., Howarth I. D., 2003, MNRAS, 345, 1223

Evans C. J., Lennon D. J., Smartt S. J., Trundle C., 2006, A\&A, 456, 623

Feast M. W., Thackeray A. D., Wesselink A. J., 1960, MNRAS, 121, 337

Fehrenbach C., 1972, A\&A, 19, 427

Fehrenbach C., Duflot M., 1982, A\&AS, 48, 409

Ferguson J. W., Alexander D. R., Allard F., Barman T., Bodnarik J. G., Hauschildt P. H., Heffner-Wong A., Tamanai A., 2005, ApJ, 623, 585

Fitzpatrick E. L., Garmany C. D., 1990, ApJ, 363, 119

Flower P. J., 1996, ApJ, 469, 355

Fuller G. M., Fowler W. A., Newman M. J., 1985, ApJ, 293, 1

Fuller J., Piro A. L., Jermyn A. S., 2019, MNRAS, 485, 3661

Fulmer L. M., Gallagher J. S., Hamann W.-R., Oskinova L. M., Ramachandran V., 2020, A\&A, 633, A164

Gaia Collaboration 2018, VizieR Online Data Catalog, p. I/345

Georgy C., et al., 2013, A\&A, 558, A103

Georgy C., Saio H., Meynet G., 2014, MNRAS, 439, L6

Glatzel W., Kiriakidis M., 1993, MNRAS, 263, 375

González-Fernández C., Dorda R., Negueruela I., Marco A., 2015, A\&A, $578, \mathrm{~A} 3$

Hainich R., et al., 2014, A\&A, 565, A27

Hainich R., Pasemann D., Todt H., Shenar T., Sand er A., Hamann W. R., 2015, A\&A, 581, A21

Hainich R., Ramachandran V., Shenar T., Sand er A. A. C., Todt H., Gruner D., Oskinova L. M., Hamann W. R., 2019, A\&A, 621, A85

Heger A., Langer N., Woosley S. E., 2000, ApJ, 528, 368

Henyey L., Vardya M. S., Bodenheimer P., 1965, ApJ, 142, 841

Herwig F., 2000, A\&A, 360, 952

Higgins E. R., Vink J. S., 2019, A\&A, 622, A50

Higgins E. R., Vink J. S., 2020, A\&A, 635, A175

Horiuchi S., Nakamura K., Takiwaki T., Kotake K., Tanaka M., 2014, MNRAS, 445, L99

Humphreys R. M., 1983, ApJ, 265, 176

Humphreys R. M., Davidson K., 1979, ApJ, 232, 409

Humphreys R. M., Weis K., Davidson K., Gordon M. S., 2016, ApJ, 825, 64

Hunter I., et al., 2007, A\&A, 466, 277

Hunter I., Lennon D. J., Dufton P. L., Trundle C., Simón-Díaz S., Smartt S. J., Ryans R. S. I., Evans C. J., 2008, A\&A, 479, 541

Iglesias C. A., Rogers F. J., 1993, ApJ, 412, 752

Iglesias C. A., Rogers F. J., 1996, ApJ, 464, 943

Indu G., Subramaniam A., 2011, A\&A, 535, A115

Itoh N., Totsuji H., Ichimaru S., Dewitt H. E., 1979, ApJ, 234, 1079

Itoh N., Hayashi H., Nishikawa A., Kohyama Y., 1996, ApJS, 102, 411

Jermyn A. S., Tout C. A., Chitre S. M., 2018, MNRAS, 480, 5427 
Kalari V. M., Vink J. S., Dufton P. L., Fraser M., 2018, A\&A, 618, A17 Kippenhahn R., Ruschenplatt G., Thomas H. C., 1980, A\&A, 91, 175

Klencki J., Nelemans G., Istrate A. G., Pols O., 2020, A\&A, 638, A55

Kochanek C. S., 2020, MNRAS, 493, 4945

Köhler K., et al., 2015, A\&A, 573, A71

Korn A. J., Becker S. R., Gummersbach C. A., Wolf B., 2000, A\&A, 353, 655

Lamers H. J. G. L. M., Fitzpatrick E. L., 1988, ApJ, 324, 279

Langanke K., Martínez-Pinedo G., 2000, Nuclear Physics A, 673, 481

Langer N., Fricke K. J., Sugimoto D., 1983, A\&A, 126, 207

Lennon D. J., 1997, A\&A, 317, 871

Levesque E. M., Massey P., Olsen K. A. G., Plez B., Josselin E., Maeder A., Meynet G., 2005, ApJ, 628, 973

Lindegren L., et al., 2016, A\&A, 595, A4

Maeder A., Meynet G., 1987, A\&A, 182, 243

Maeder A., Meynet G., 2000, A\&A, 361, 159

Mahy L., et al., 2020, A\&A, 634, A118

Massey P., Evans K. A., 2016, ApJ, 826, 224

Massey P., Olsen K. A. G., 2003, AJ, 126, 2867

Meynet G., Maeder A., 1997, A\&A, 321, 465

Moe M., Di Stefano R., 2017, ApJS, 230, 15

Neugent K. F., Massey P., Skiff B., Drout M. R., Meynet G., Olsen K. A. G., 2010, ApJ, 719, 1784

Neugent K. F., Massey P., Skiff B., Meynet G., 2012, ApJ, 749, 177

Nugis T., Lamers H. J. G. L. M., 2000, A\&A, 360, 227

Oda T., Hino M., Muto K., Takahara M., Sato K., 1994, Atomic Data and Nuclear Data Tables, 56, 231

Ohnaka K., Driebe T., Hofmann K. H., Weigelt G., Wittkowski M., 2008, A\&A, 484, 371

Paxton B., Bildsten L., Dotter A., Herwig F., Lesaffre P., Timmes F., 2011, ApJS, 192, 3

Paxton B., et al., 2013, ApJS, 208, 4

Paxton B., et al., 2015, ApJS, 220, 15

Paxton B., et al., 2018, ApJS, 234, 34

Pietrzyński G., et al., 2013, Nature, 495, 76

Potekhin A. Y., Chabrier G., 2010, Contributions to Plasma Physics, 50, 82

Potter A. T., Tout C. A., Eldridge J. J., 2012, MNRAS, 419, 748

Ramachandran V., Hainich R., Hamann W. R., Oskinova L. M., Shenar T., Sander A. A. C., Todt H., Gallagher J. S., 2018a, A\&A, 609, A7

Ramachandran V., Hamann W. R., Hainich R., Oskinova L. M., Shenar T., Sander A. A. C., Todt H., Gallagher J. S., 2018b, A\&A, 615, A40

Ramachandran V., et al., 2019, A\&A, 625, A104

Ramírez-Agudelo O. H., et al., 2015, A\&A, 580, A92

Ramírez-Agudelo O. H., et al., 2017, A\&A, 600, A81

Rogers F. J., Nayfonov A., 2002, ApJ, 576, 1064

Salpeter E. E., 1954, Australian Journal of Physics, 7, 373

Sana H., et al., 2012, Science, 337, 444

Sanduleak N., 1970, Contributions from the Cerro Tololo Inter-American Observatory, 89

Sanyal D., Langer N., Szécsi D., -C Yoon S., Grassitelli L., 2017, A\&A, 597, A71

Saumon D., Chabrier G., van Horn H. M., 1995, ApJS, 99, 713

Schneider F. R. N., et al., 2018, A\&A, 618, A73

Schootemeijer A., Langer N., 2018, A\&A, 611, A75

Schootemeijer A., Langer N., Grin N. J., Wang C., 2019, A\&A, 625, A132

Shaviv G., Salpeter E. E., 1973, ApJ, 184, 191

Shenar T., et al., 2016, A\&A, 591, A22

Shenar T., et al., 2017, A\&A, 598, A85

Shenar T., et al., 2019, A\&A, 627, A151

Shenar T., Gilkis A., Vink J. S., Sana H., Sander A. A. C., 2020, A\&A, 634, A79

Smartt S. J., Eldridge J. J., Crockett R. M., Maund J. R., 2009, MNRAS, 395, 1409

Smith N., 2019, MNRAS, 489, 4378

Stock J., Osborn W., Ibanez M., 1976, A\&AS, 24, 35

Stothers R. B., Chin C.-W., 1991, ApJ, 374, 288

Tabernero H. M., Dorda R., Negueruela I., González-Fernández C., 2018, MNRAS, 476, 3106
Tehrani K. A., Crowther P. A., Bestenlehner J. M., Littlefair S. P., Pollock A. M. T., Parker R. J., Schnurr O., 2019, MNRAS, 484, 2692

Timmes F. X., Swesty F. D., 2000, ApJS, 126, 501

Torres G., 2010, AJ, 140, 1158

Tramper F., et al., 2015, A\&A, 581, A110

Tramper F., Sana H., de Koter A., 2016, ApJ, 833, 133

Trundle C., Lennon D. J., 2005, A\&A, 434, 677

Trundle C., Lennon D. J., Puls J., Dufton P. L., 2004, A\&A, 417, 217

Trundle C., Dufton P. L., Hunter I., Evans C. J., Lennon D. J., Smartt S. J., Ryans R. S. I., 2007, A\&A, 471, 625

Ulmer A., Fitzpatrick E. L., 1998, ApJ, 504, 200

Urbaneja M. A., Kudritzki R. P., Gieren W., Pietrzyński G., Bresolin F., Przybilla N., 2017, AJ, 154, 102

Vink J. S., de Koter A., Lamers H. J. G. L. M., 2001, A\&A, 369, 574

Vink J. S., Brott I., Gräfener G., Langer N., de Koter A., Lennon D. J., 2010, A\&A, 512, L7

Walmswell J. J., Tout C. A., Eldridge J. J., 2015, MNRAS, 447, 2951

Wenger M., et al., 2000, A\&AS, 143, 9

Wolf B., 1972, A\&A, 20, 275

Woosley S. E., 2019, ApJ, 878, 49

Yoon S.-C., 2017, MNRAS, 470, 3970

de Jager C., Nieuwenhuijzen H., van der Hucht K. A., 1988, A\&AS, 72, 259 